I Y $\varepsilon$

الميزان التجاري للمياه الافتراضية لأهم الحاصلات الزراعية المصرية

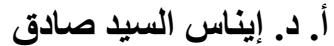

أستاذ الاقتصاد الزر أعي بكلية الزر اعة العبد - جامعة الفيوم

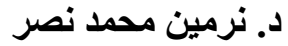

باحث مساعد بمعهد بحوث الاقتصاد الزر اعي نصيز

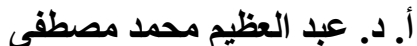

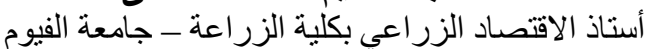

أ. د. وحيد محمد البولوني لزي لئهي

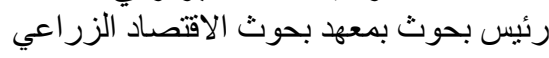

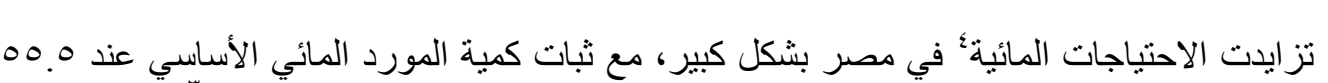

تمهيد:

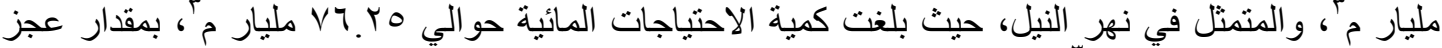

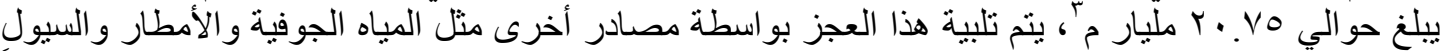

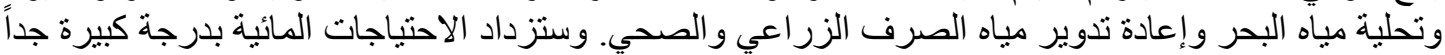

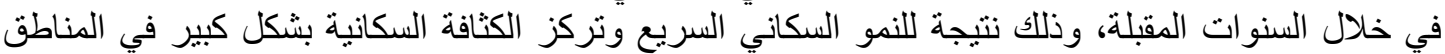

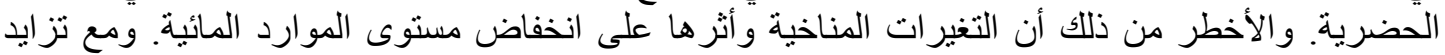

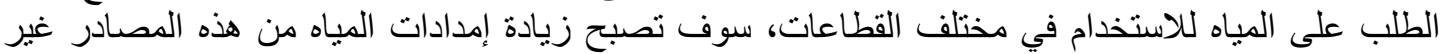

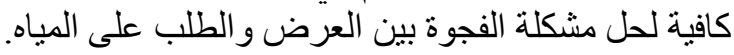

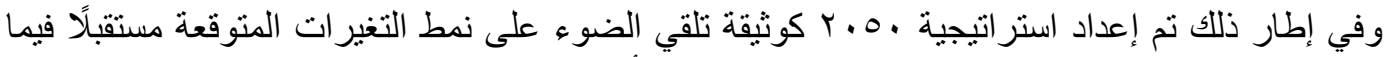

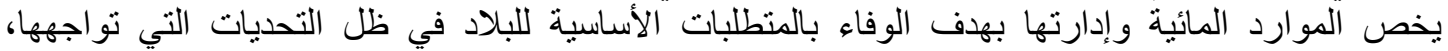

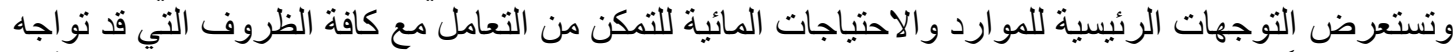

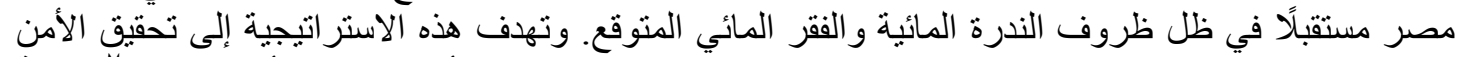

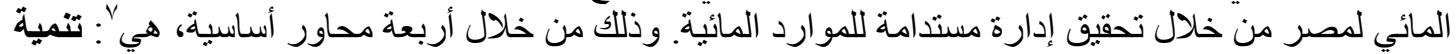

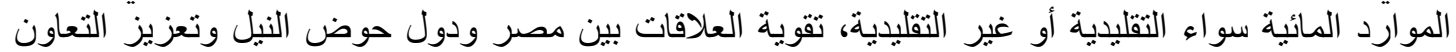

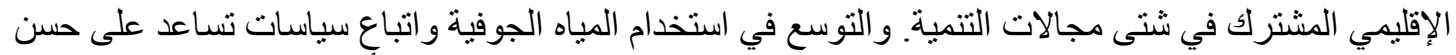

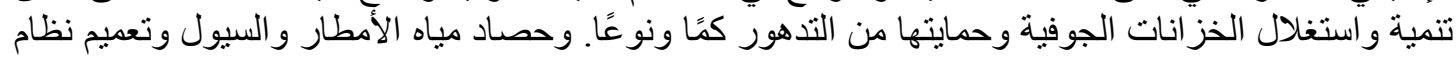

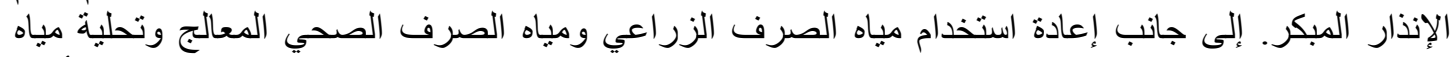

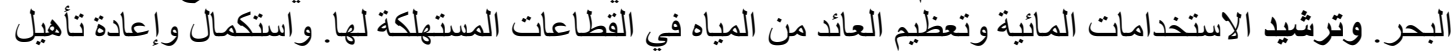

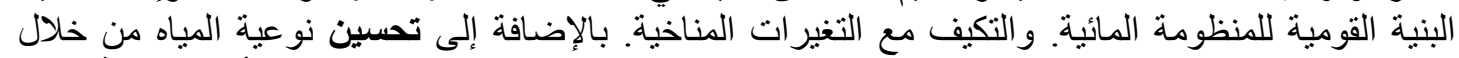

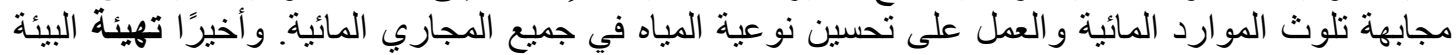
الملائمة للإدارة المتكاملة للموارد المارد المائية.

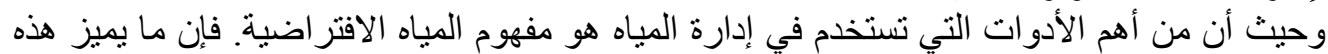

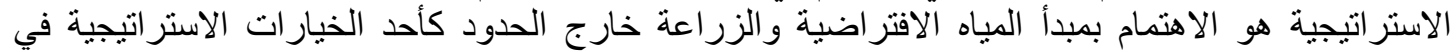

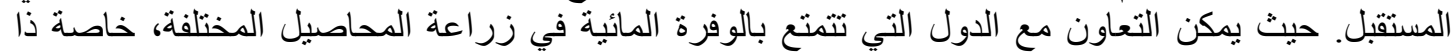

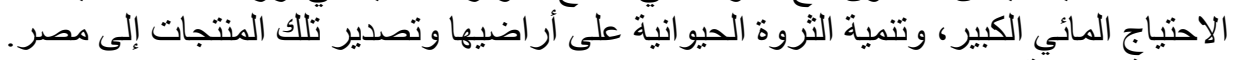
المشكلة البحثية: المئية

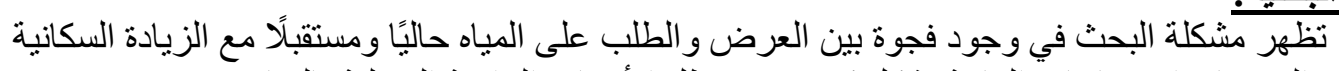

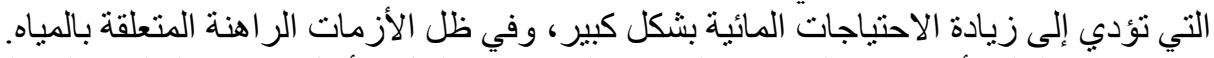

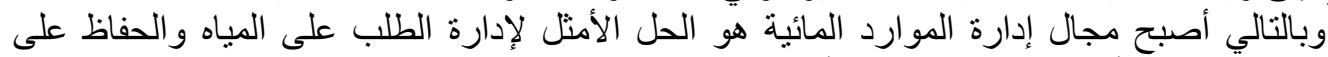

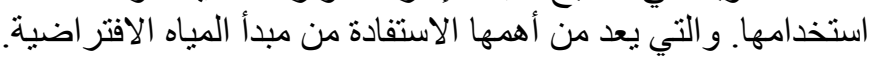
أهداف البحث:

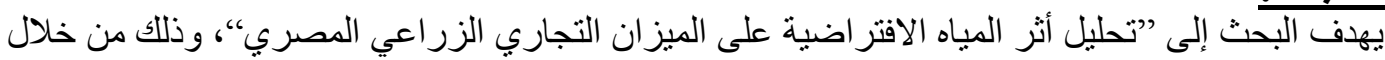

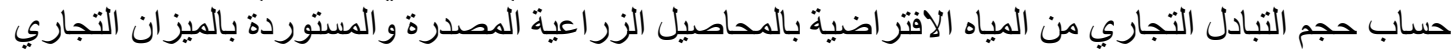
الزر اعي المصري. وتقدير البصمة المائية لها. الطريقة البحثية ومصدادر البيانات: البئية

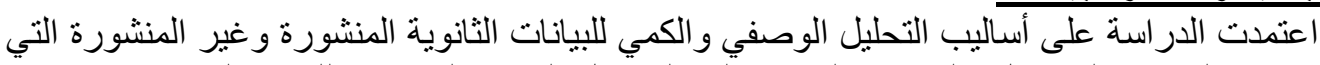

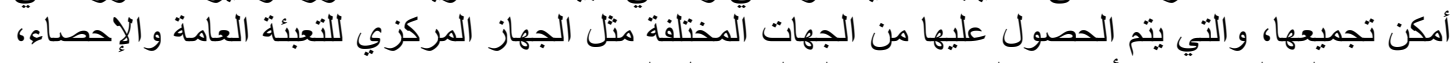

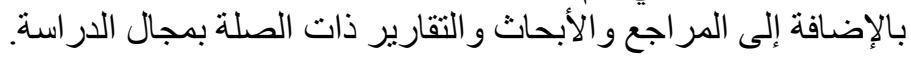

Fayoum J. Agric. Res. \& Dev., Vol. 34, No.2 July, 2020 
وقد تم تقدير محتوى المياه الافتراضية للمحاصيل المصدرة و المستوردة بالميزان التجاري الزراعي

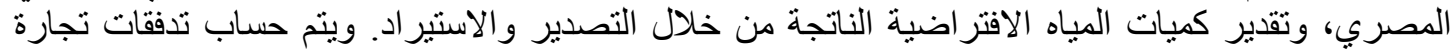

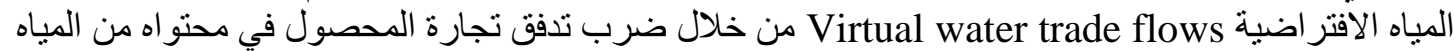

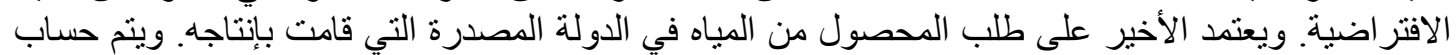

$$
\mathrm{VWT}=\mathrm{CT} * \mathrm{SWD}
$$

تجارة المياه الافتر اضية كما يلي الاضئ:

حيث:

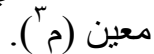
CT تجارة المحصول من الدولة المصدرة إلى الدولة المستوردة (طن). SWD : طلب المحصول من المياه في الدولة المصدرة (مّآلإطن).

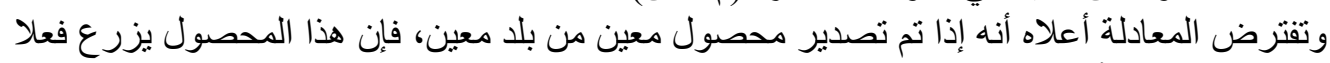

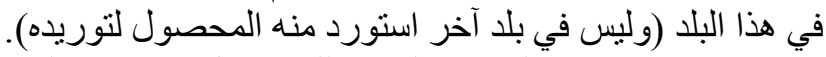

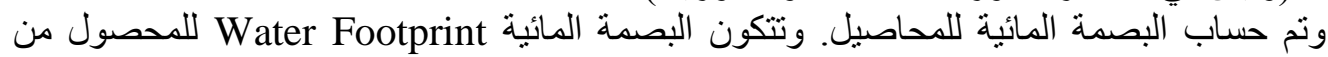

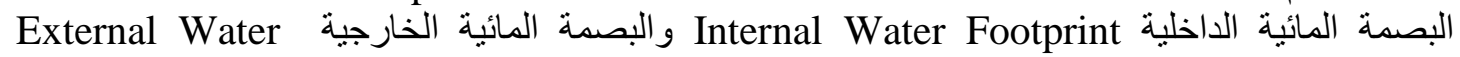
Footprint

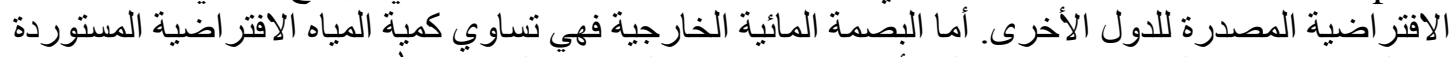

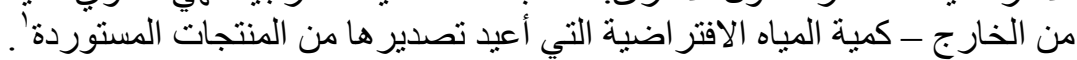

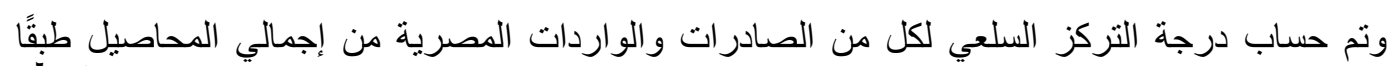

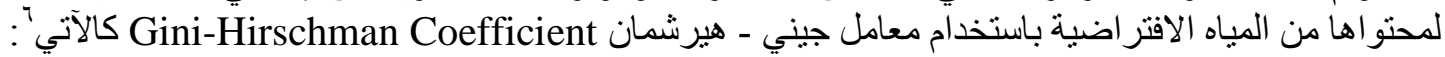

$$
C i=\sqrt{\sum\left(\frac{x i j}{x i}\right)^{2}} * 100
$$

حيث: Ci تمثل معامل التركيز السلعي لكمية أو قيمة الصادرات.

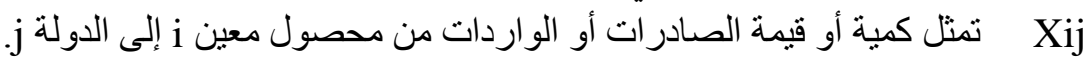

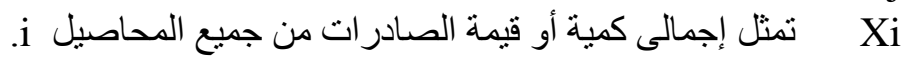

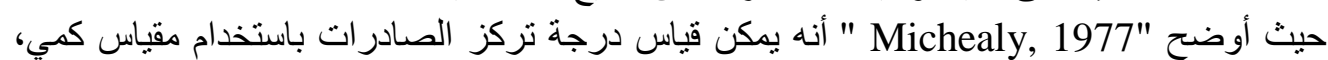

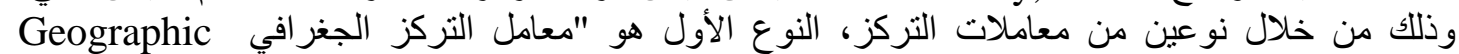
Concentration Coefficient

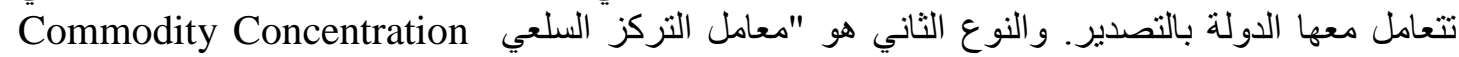
Coefficient

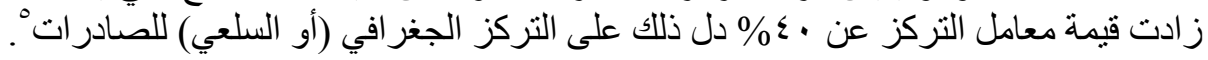

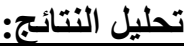

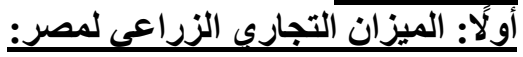

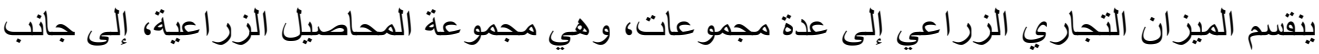

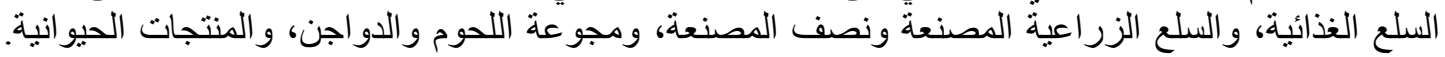

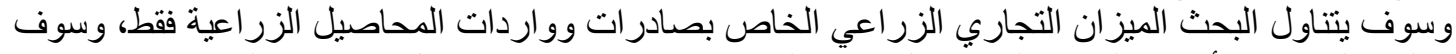

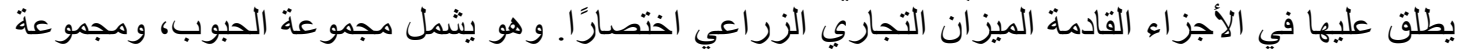

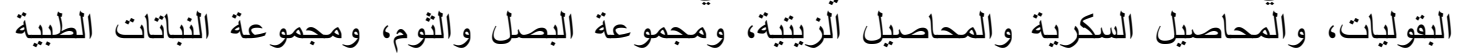

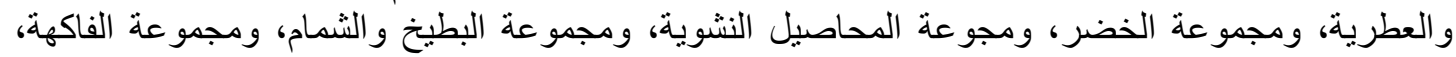

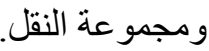

ا ـ الأهمية النسبية للميزان التجاري الزراعي بجمهورية مصر العربية:

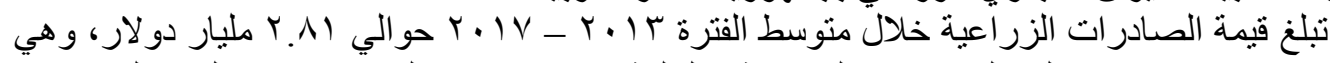

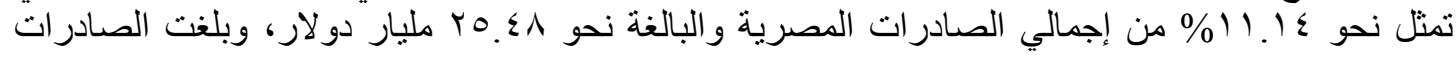

Fayoum J. Agric. Res. \& Dev., Vol. 34, No.2 July, 2020 


\section{Y T}

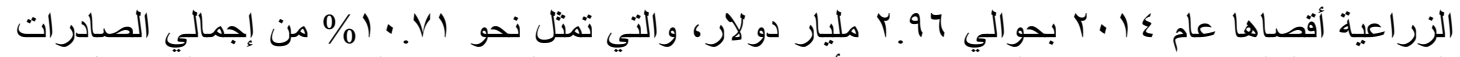

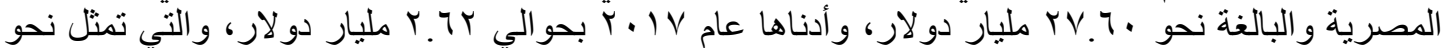

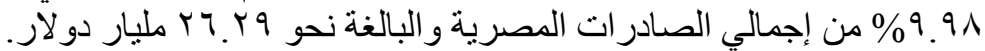

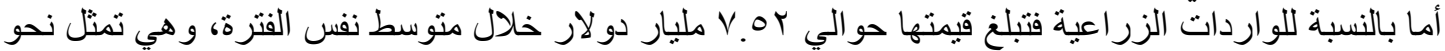

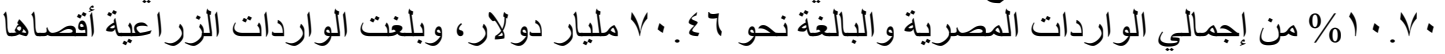

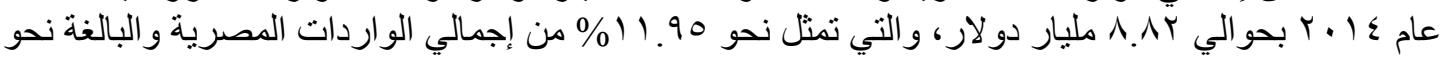

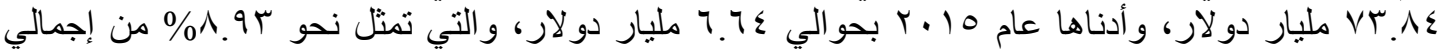

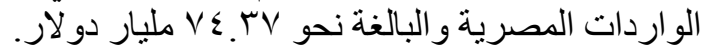

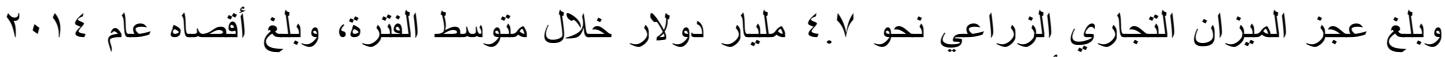

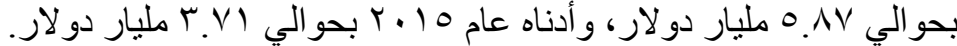

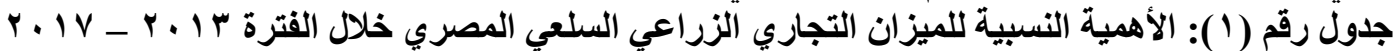
بالمليون دولار

\begin{tabular}{|c|c|c|c|c|c|c|c|}
\hline عجز الميزان التجاري & $\%$ & الزار الزداتة & الواردات الكلبة & $\%$ & الزرادراتية الصية & الصادرات الكلية & السنو ات \\
\hline OYI $\varepsilon_{-}$ & IT.YE & 1.99 & $77) \wedge$. & $9.9 \leq$ & 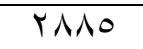 & rq.11 & $r .1 T$ \\
\hline $0 \wedge 70_{-}$ & 11.90 & AᄉYI & 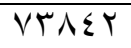 & $1 . . V 1$ & Y907 & rVT.. & Y. IE \\
\hline rVIT_ & 1.94 & $77 \leqslant 1$ & $V \leqslant T V \leqslant$ & Tr.T & rqr. & YIqAV & $r .10$ \\
\hline รイルน & 9.71 & 79.5 & VITOV & $11 . V \varepsilon$ & rาะI & YrO. Y & T.17 \\
\hline$\varepsilon \leqslant 9 V_{-}$ & $1 . V$. & VIY. & 77070 & 9.91 & & イฯイトT & $r .1 \mathrm{~V}$ \\
\hline$\varepsilon V \mid \cdot-$ & $1 . . V \cdot$ & VOIV & $V \cdot \Sigma 7 r$ & $11.1 \varepsilon$ & $r \wedge \cdot V$ & roミVA & المتوسط \\
\hline
\end{tabular}

المصدر: جمعت وحسبت من: الجهاز المركزي للتعبئة العامة والإحصاء، النشرة السنوية للتجارة الخارجية، القاهرة،

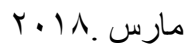

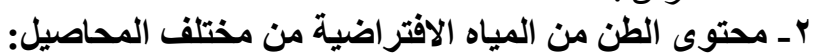

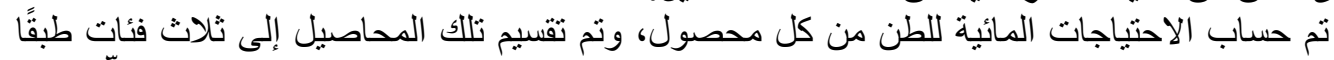

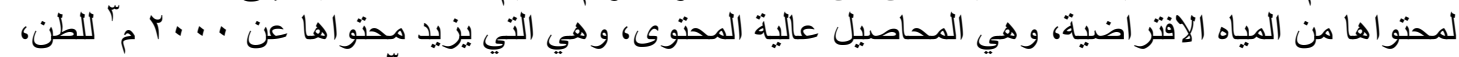

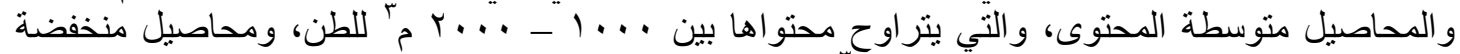

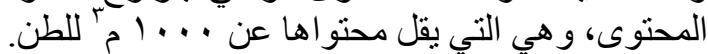

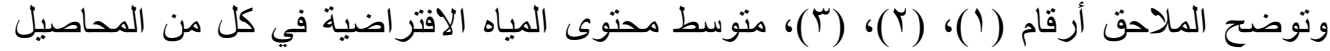

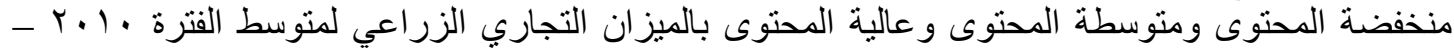

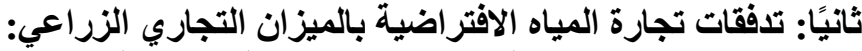

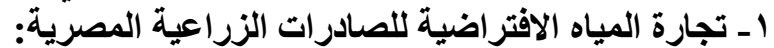

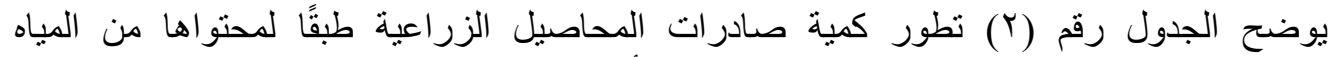

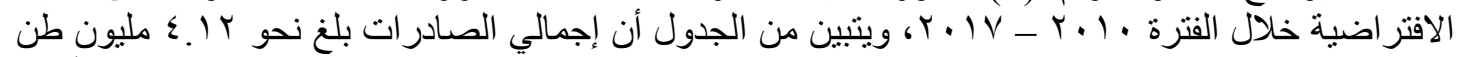

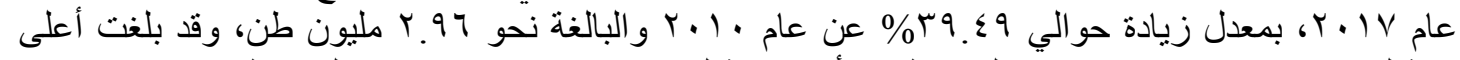

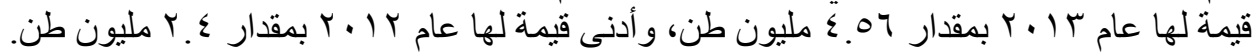

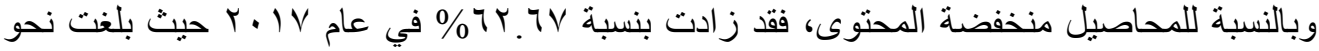

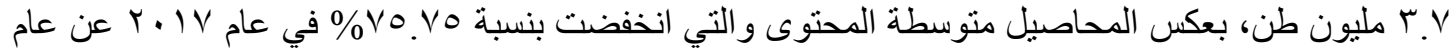

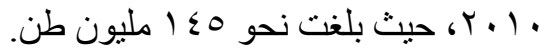

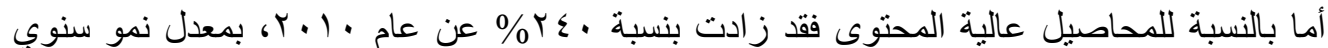

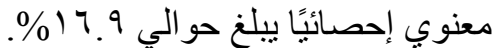

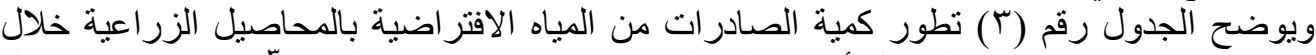

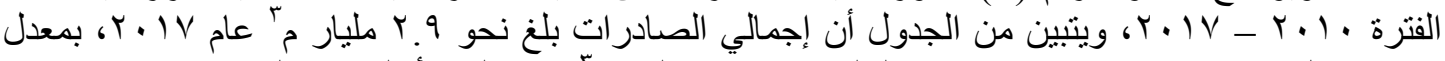

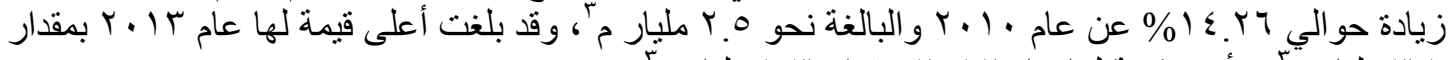

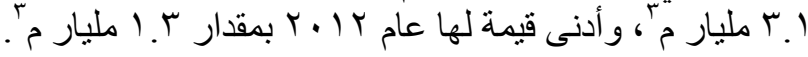

Fayoum J. Agric. Res. \& Dev., Vol. 34, No.2 July, 2020 
I TV

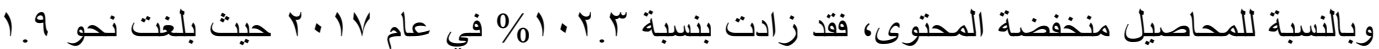

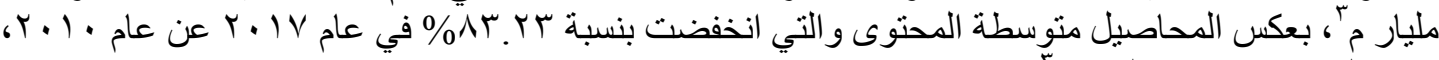

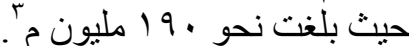

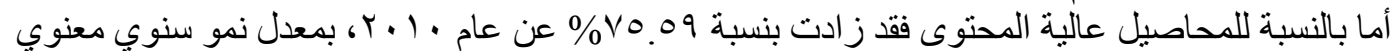

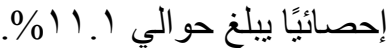
جدول رقم (r): تطور كمية صادرات المحاصيل الزراعية المصرية طبقًا لمحتواها من المياه الافتراضية خلال الكمية بالألف طن المن المبان

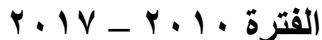

\begin{tabular}{|c|c|c|c|c|}
\hline إجمالي الصادرات & منخفضة المحتوى & متوسطة المحتوى & عالية المحتوى & السنوات \\
\hline r900 & TYVV & 091 & $\Lambda$. & $r .1$. \\
\hline rrAl & TIYA & $V 7$ & $V V$ & $r .11$ \\
\hline rrqq & Y1.T & 191 & 90 & T.IT \\
\hline$\{00 \wedge$ & rNIT & $0 \wedge 7$ & 17. & $r .1 T$ \\
\hline$\Gamma \cdot \varepsilon \varepsilon$ & TVAT & TKS & $10 \mathrm{~V}$ & $r . I \varepsilon$ \\
\hline$r \leqslant 70$ & $\Gamma \cdot \Lambda \tau$ & TYZ & 100 & $r .10$ \\
\hline 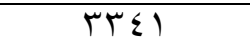 & $r \cdot Y r$ & $1 \leqslant$. & 179 & T.17 \\
\hline$\varepsilon / Y Y$ & $r V_{\cdot} \varepsilon$ & $1 \leqslant 0$ & TVY & $T . I V$ \\
\hline$r r 97$ & rqAV & Y7I & $1 \leqslant V$ & المتوسط \\
\hline $\begin{array}{c}r . V \\
(1.097)\end{array}$ & $\begin{array}{c}\varepsilon . \wedge \\
(\nvdash . \vee q \wedge)\end{array}$ & $\begin{array}{c}9.7- \\
-(.710)\end{array}$ & $\begin{array}{c}17.9 \\
*(\leqslant 9.91)\end{array}$ & نمو \% \\
\hline
\end{tabular}

حيث: الأرقام الموجودة بين الأقو اس أسفل قيمة معدل النمو بالجدول تثشير إلى قيمة (F) المحسوبة.

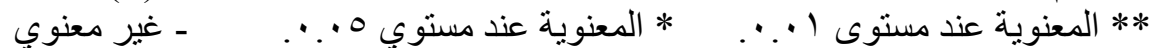

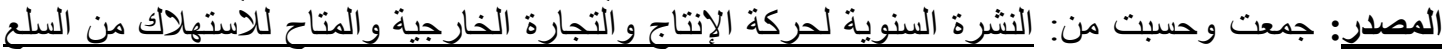

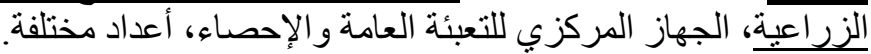

جدول رقم (ب): تطور كمية صادرات المياه الافتراضية من المحاصيل الزراعية طبقًا لمحتو اها من المياه

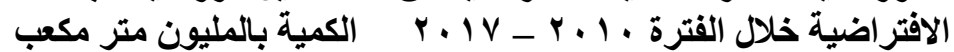

\begin{tabular}{|c|c|c|c|c|}
\hline إجمالي الصادرات & منخفضة المحتوى & متوسطة المحتوى & عالية المحتوى & السنوات \\
\hline TOIV & 901 & 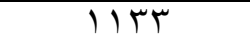 & $\sum Y \tau$ & $r \cdot 1$. \\
\hline $171 \varepsilon$ & $1 K \leqslant 7$ & 10 & rAT & $r .11$ \\
\hline $1 Y 97$ & $\wedge \wedge 0$ & $T \cdot Y$ & YI. & $T . I T$ \\
\hline TIYT & 1919 & $\vee \wedge \wedge$ & $\varepsilon 19$ & $r .1 T$ \\
\hline $17 \Lambda \varepsilon$ & $1 . V \pi$ & $17 \varepsilon$ & $\varepsilon \leqslant V$ & $T \cdot 1 \varepsilon$ \\
\hline 1914 & $1 \% 9 \varepsilon$ & r91 & rq1 & $r .10$ \\
\hline THIT & $10 \leqslant 7$ & 191 & 079 & $r .17$ \\
\hline YAVT & $19 \mathrm{~K}$ & 19. & $V \leqslant \Lambda$ & $T . I V$ \\
\hline rIVT & $1 T 0 V$ & rᄉr & $\varepsilon r V$ & المتوسط \\
\hline $\begin{array}{c}\varepsilon \\
-(\cdot V \cdot r)\end{array}$ & $\begin{array}{c}V . \wedge \\
(\varepsilon . Y \mu T)\end{array}$ & $\begin{array}{c}1 \cdot . r_{-} \\
(\cdot . \Delta V V)\end{array}$ & $\begin{array}{c}11.1 \\
* *(0 . \vee 07)\end{array}$ & معدل النمو \% \\
\hline
\end{tabular}

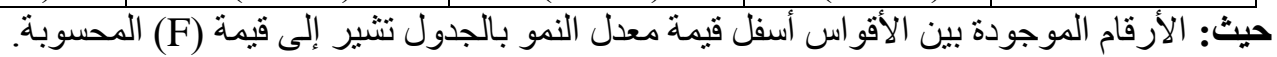

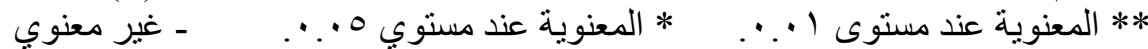

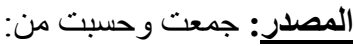
ــ الجهاز المركزي للتعبئة العامة و الإحصاء، النشرة السنوية لحركة الإنتاج و التجارة الخارجية والدتاح للاستهلاك من السلع الزر اعية، القاهرة، أعداد مختلفة.

- الجهاز المركزي للتعبئة العامة واعة الإحصاء، النشرة السنوية لإحصاء الري والموارد المائية، القاهرة، أعداد مختلفة.

Fayoum J. Agric. Res. \& Dev., Vol. 34, No.2 July, 2020 
IY

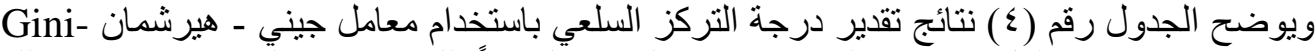
Hirschman Coefficient

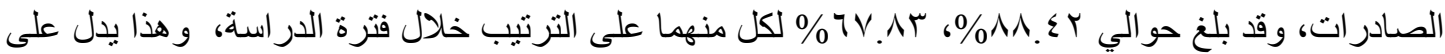

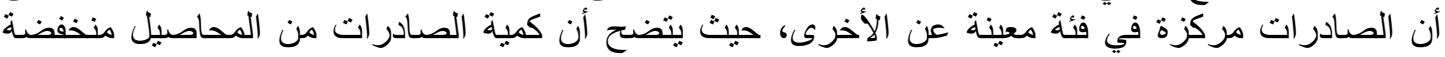

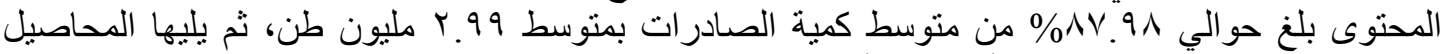

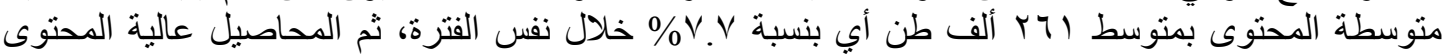

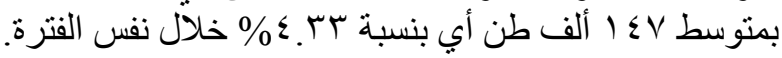

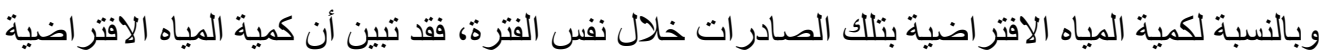

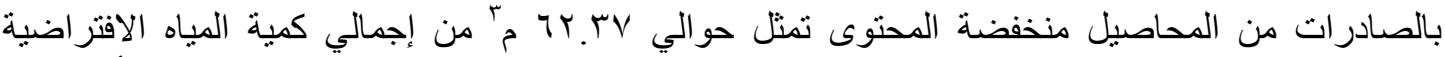

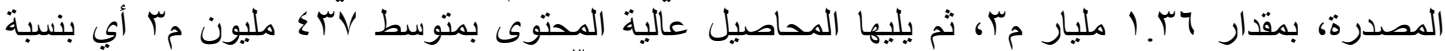

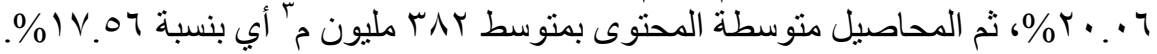

جدول رقم (؛): تقدير معامل جيني - هيرشمان للتركز السلعي لكمية الصادرات وكمية المياه الافتراضية

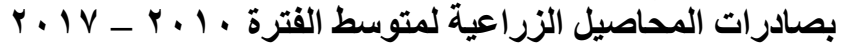

\begin{tabular}{|c|c|c|c|c|}
\hline \multicolumn{2}{|c|}{ كمية المياه الافتر اضية بالصادرات } & \multicolumn{2}{|c|}{ كمية الصادرات } & \multirow{2}{*}{ الفئة } \\
\hline$\%$ & متوسط & $\%$ & متوسط & \\
\hline$T \cdot . T$ & $\varepsilon r V$ & $\varepsilon . \Gamma$ & $1 \leqslant V$ & عالية المحتوى \\
\hline $1 V .07$ & TAY & $\vee . \vee \cdot$ & YTI & متوسطة المحتوى \\
\hline Tr.rV & ITOV & 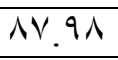 & rqAV & منخفضة المحتوى \\
\hline $1 \cdots$ & YIVT & $1 \cdots$ & r & إجمالي \\
\hline \multicolumn{2}{|c|}{ 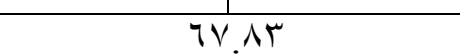 } & \multicolumn{2}{|c|}{$\lambda \wedge . \varepsilon Y$} & معامل جيني - هيرشمان \\
\hline
\end{tabular}

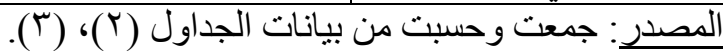

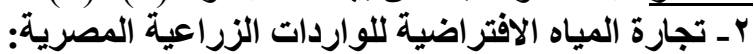

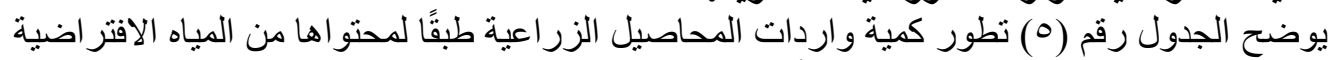

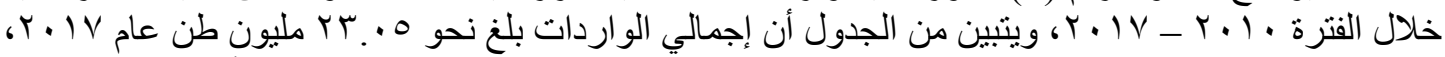

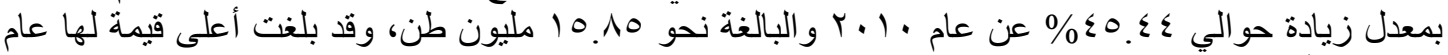

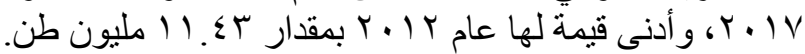

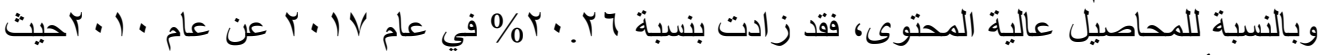

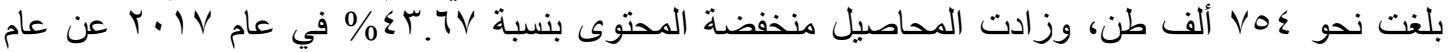

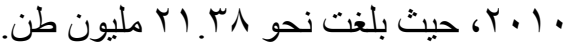

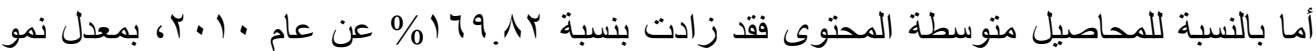

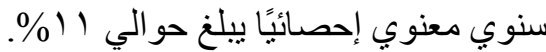

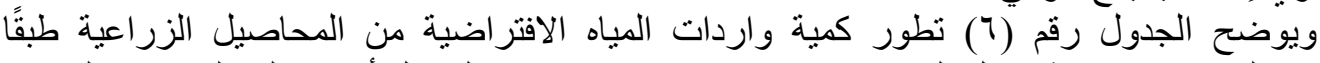

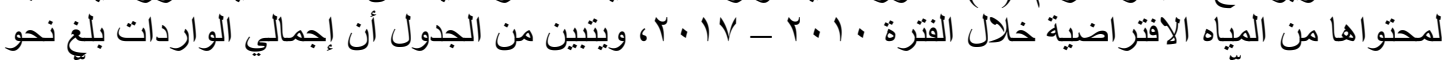

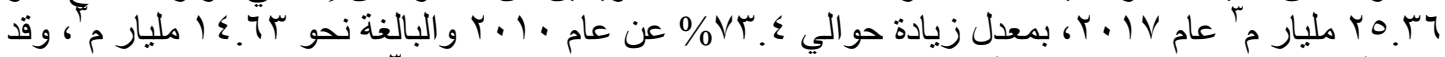

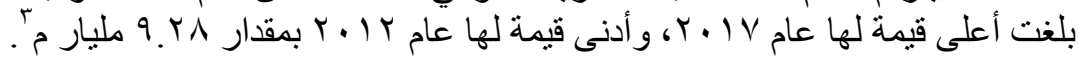

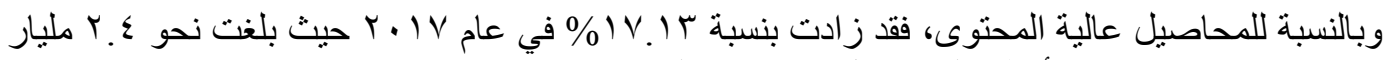

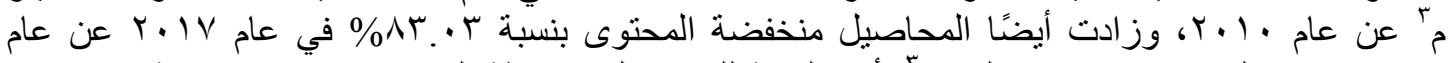

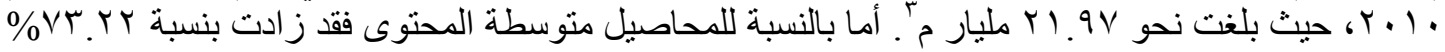

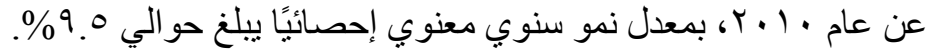

Fayoum J. Agric. Res. \& Dev., Vol. 34, No.2 July, 2020 
1 1 19

جدول رقم (•): تطور كمية الواردات من المحاصيل الزراعية طبقًا لمحتواها من المياه الافتراضية خلال الفترة

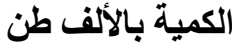

\begin{tabular}{|c|c|c|c|c|}
\hline إجمالي الواردات & منخفضة المحتوى & متوسطة المحتوى & عالية المحتوى & السنوات \\
\hline loरEV & $I \leqslant \wedge \wedge r$ & Tr & TYV & $r .1$. \\
\hline$r .9 \leq q$ & $1 V \cdot 19$ & 011 & Tr & $r .11$ \\
\hline $11 \leqslant Y 0$ & I. YAr & אדצ & VA. & $r \cdot 1 r$ \\
\hline 1717 & $|\leqslant| 1$ & $7 \leqslant \varepsilon$ & $100 \%$ & $r .1 T$ \\
\hline I $\leqslant V \Psi \varepsilon$ & IEY. & $\{71$ & 79 & r. IE \\
\hline IVT. Y & 1ד & $0 . V$ & TrV & $r .10$ \\
\hline IGYTY & $1 V \leq \leqslant \Lambda$ & $V \cdot V$ & 1.87 & $r .17$ \\
\hline$r \mu \cdot \varepsilon \Lambda$ & Y\ハ人 & $9 / r$ & Vos & $r . I V$ \\
\hline IVTE & IOVYY & 000 & $1.7 V$ & المتوسط \\
\hline $\begin{array}{c}\varepsilon \\
(1 . \leqslant \circ 4)\end{array}$ & $\begin{array}{c}\varepsilon . \wedge \\
-(r .774)\end{array}$ & $\begin{array}{c}11 \\
* *(1 \cdot .9 \vee \cdot)\end{array}$ & $\begin{array}{c}I Y_{-} \\
-\left(\cdot \Sigma r^{\prime}\right)\end{array}$ & عدل النمو \% \\
\hline
\end{tabular}

حيث: الأرقام الموجودة بين الأقو اس أسفل قيمة معدل النمو بالجدول تثشير إلى قيمة (F) المحسوبة.

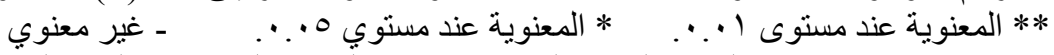

المصدر: جمعت وحسبت من: النشرة السنوية لحركة الإنتاج و التجارة الخارجية و المتاح للاستهلاك من السلع الزربة اعية، الجهاز المركزي للتعبئة العامة و الإحصاء، أعداد مختوة العتلفة. جدول رقم (†): تطور كمية واردات المياه الافتراضية من المحاصيل الزراعية طبقًا لمحتو اها من المياه

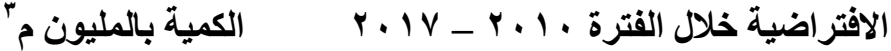

\begin{tabular}{|c|c|c|c|c|}
\hline إجمالي الواردات & منخفضة المحتوى & متوسطة المحتوى & عالية المحتوى & السنوات \\
\hline $1 \leq 7 T V$ & $I r \ldots r$ & OVo & $r \cdot \leqslant q$ & $r .1$. \\
\hline$r \ldots .09$ & 11VTs & $\varepsilon \wedge 9$ & VArT & $r+11$ \\
\hline 9 9र11 & $79 \leqslant \varepsilon$ & TTV & $19 V 1$ & $T . / T$ \\
\hline 1071. & 1.110 & $\Lambda \leqslant 1$ & rqo & $r .1 T$ \\
\hline IYVOO & $1 \cdot Y 71$ & $T \leqslant Y$ & INOY & $r \cdot 1 \varepsilon$ \\
\hline $1 \leqslant V 17$ & ITIYA & Tro & $97 \leqslant$ & $r .10$ \\
\hline$r \cdot 77 r$ & $1700 \mathrm{~V}$ & ᄉTร & $r Y \leqslant 1$ & $r .17$ \\
\hline TOMTr & F197V & 997 & $r \xi \ldots$ & T.IV \\
\hline $177 \leqslant r$ & 1Yq40 & TVO & T.Tr & المتوسط \\
\hline $\begin{array}{c}7.1 \\
(1 . \vee 9 \Lambda)\end{array}$ & $\begin{array}{c}9.4 \\
(\varepsilon .9 \cdot 1)\end{array}$ & $* \begin{array}{c}9.0 \\
*(7.19 \leq)\end{array}$ & $\begin{array}{c}V . \varepsilon_{-} \\
(.07 r)\end{array}$ & $\begin{array}{c}\text { معدل النمو } \\
\text { \% }\end{array}$ \\
\hline
\end{tabular}

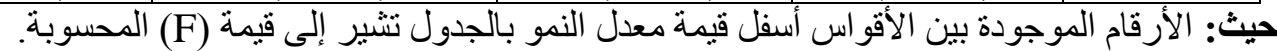

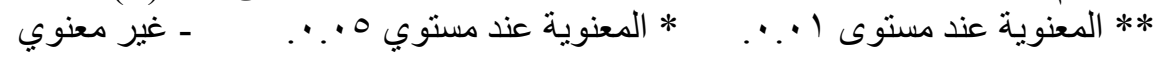

المصدر: جمعت وحسبت من: المند - الجهاز المركزي للتعبئة العامة والإحصاء، النشرة السنوية لحركة الإنتاج والتجارة الخارجية والمناح للاستهلاك من السلع الزر اعية، القاهرة، أعداد مختلفة.

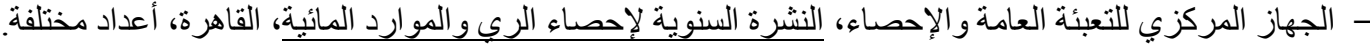

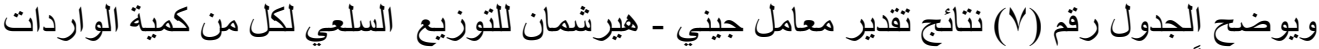

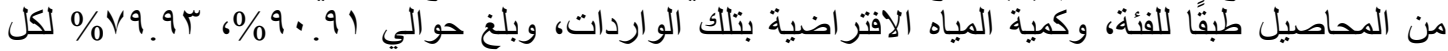

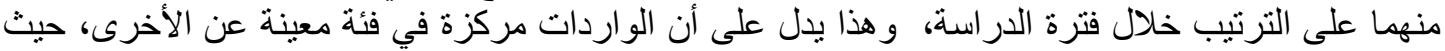

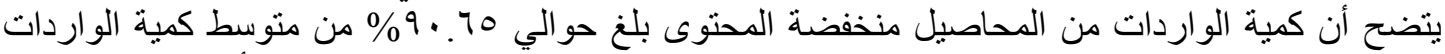

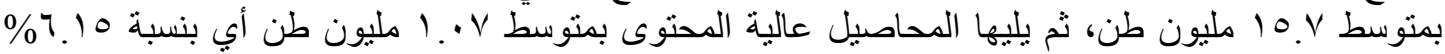

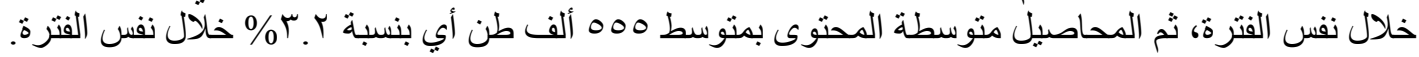

Fayoum J. Agric. Res. \& Dev., Vol. 34, No.2 July, 2020 
$1 \%$.

وبالنسبة لكمية المياه الافتر اضية بتلك الواردات خلال نفس الفترة، فقد تبين أن كمية المياه الافتر اضية

بالواردات من المحاصيل منخفضة المحتوى تمثل حوالي من من إجمالي كمية المياه الافتراضية

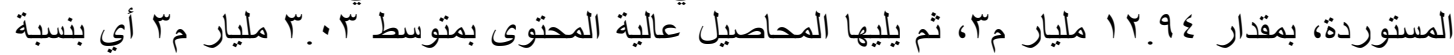

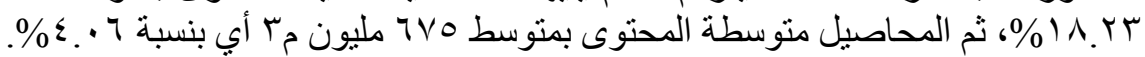

جدول رقم (V): تقدير معامل جيني - هيرشمان للتركز السلعي لكمية الواردات وكمية المياه الافتراضية

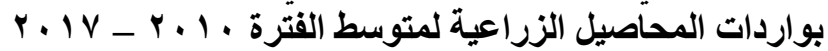

\begin{tabular}{|c|c|c|c|c|}
\hline \multicolumn{2}{|c|}{ كمية المياه الافتر اضبية بالواردات } & \multicolumn{2}{|c|}{ كمية الواردات } & \multirow{2}{*}{ الفئة } \\
\hline$\%$ & متوسط & $\%$ & متوسط & \\
\hline IN.YT & r.r & 7.10 & $1.7 V$ & عالية المحتوى \\
\hline$\varepsilon .7$ & $7 \times 0$ & r.r. & 000 & متوسطة المحتوى \\
\hline VV.VY & I T940 & $9 . .70$ & IOVYY & منخفضة المحتوى \\
\hline $1 \cdots$ & $177 \leqslant r$ & $1 \cdots$ & IVTE & إجمالي \\
\hline \multicolumn{2}{|c|}{89.94} & \multicolumn{2}{|c|}{$9 \cdot .91$} & معامل جيني - هيرشمان \\
\hline
\end{tabular}

المصدر: جمعت وحسبت من الجداول أرقام (0)، (7) (7).

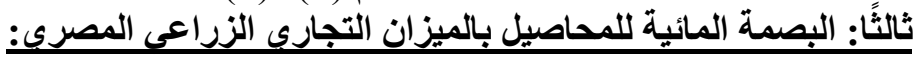

يوضح الجدول رقم (^) التطور السنوي للبصمة المائية للمحاصيل منخفضة المحتوى خلال الفترة

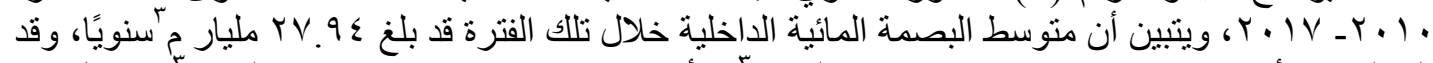

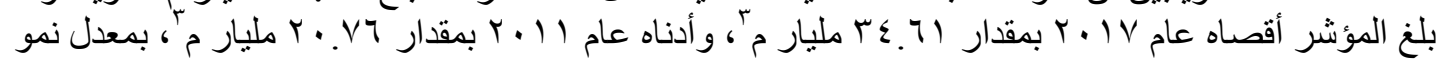

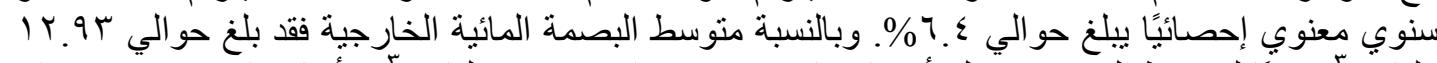

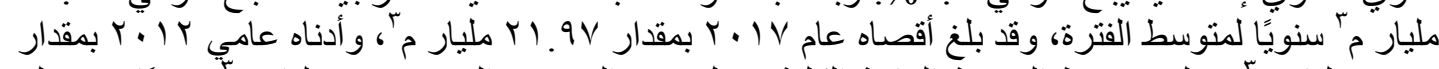

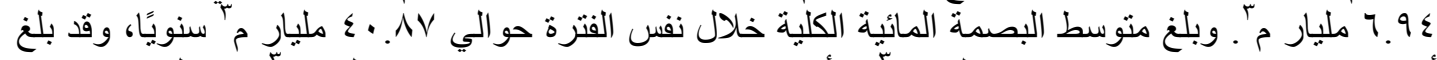

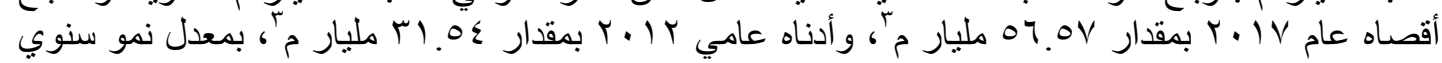

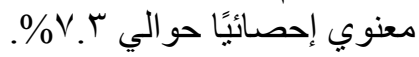

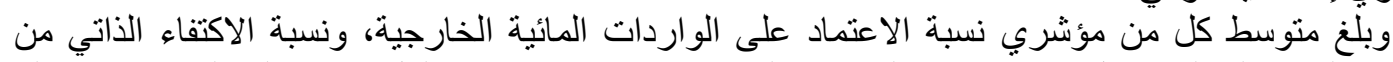

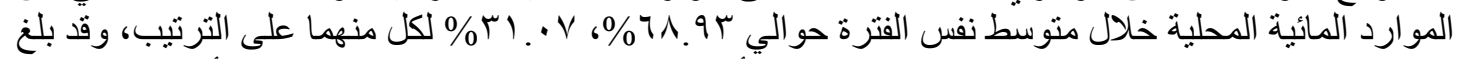

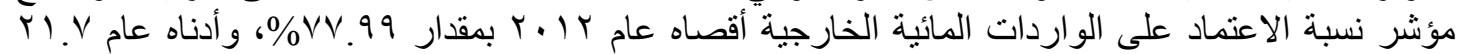

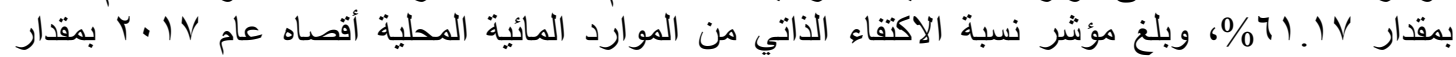

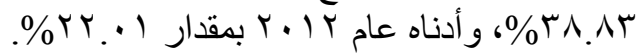

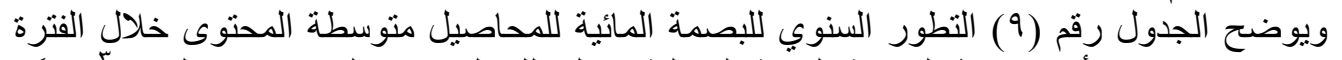

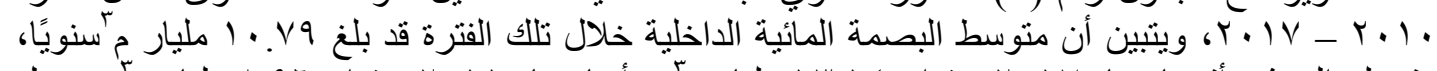

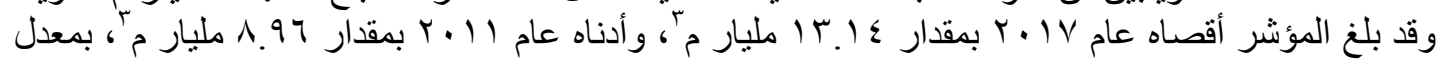

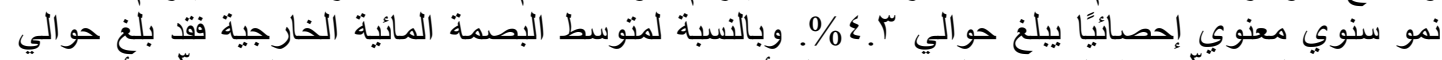

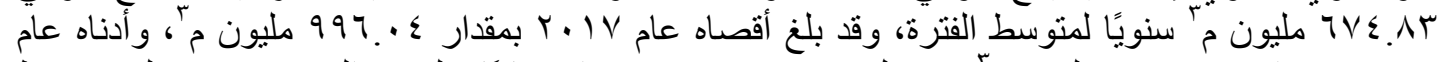

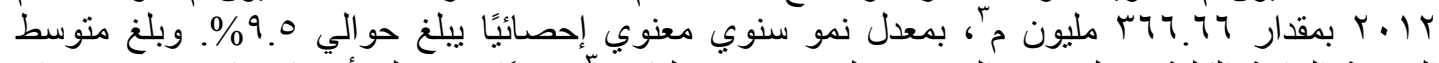

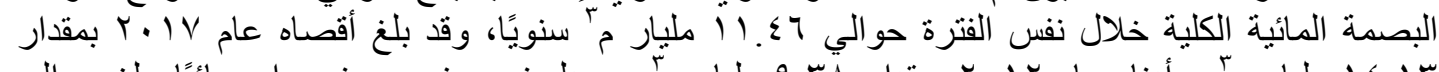

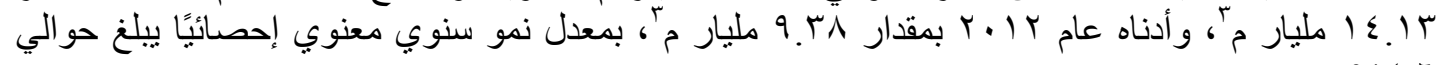


$|r|$

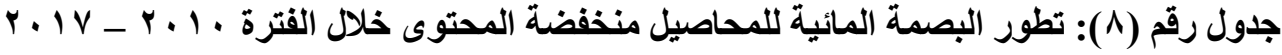

\begin{tabular}{|c|c|c|c|c|c|}
\hline \multirow{2}{*}{ نسبة الاكتفاء الأتية } & \multirow{2}{*}{ الوبة الاعتماد على الخائية } & \multicolumn{3}{|c|}{ البصمة المائية } & \multirow{2}{*}{ السنوات } \\
\hline & & الكلية & الخارجية & الاخلية & \\
\hline rY.90 & $7 V .0$ & 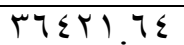 & IY... ${ }^{\prime}$ & $r \leqslant \leqslant 19.0 \mathrm{~V}$ & $r \cdot 1$. \\
\hline r. & $7 r . \wedge 9$ & $r Y \leqslant q \leqslant . Y$. & IIVTE.P & $T \cdot V 7 . .1 V$ & $r .11$ \\
\hline YY. ${ }^{\prime}$ & $V \vee .99$ & Mos $10 \leqslant \Lambda$ & $79 \leqslant r .09$ & $r\{\neg \ldots, \wedge \wedge$ & $r .1 T$ \\
\hline TV.9V & $V Y_{.} \cdot T$ & rیq11.0. & $1 . \wedge \wedge 0 . \mu \mu$ & YA.Y7.IV & $T .1 T$ \\
\hline Y7.01 & $V T . \varepsilon r$ & 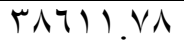 & $1 . Y 71.1 Y$ & YATO..70 & $r \cdot 1 \varepsilon$ \\
\hline 51.19 & $7 \wedge . \wedge 1$ & $\varepsilon Y \cdot \wedge \Lambda . T \leqslant$ & ITIYV.Aq & 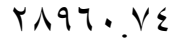 & $r \cdot 10$ \\
\hline rY.Aq & $7 V .11$ & $0 . r \leqslant .0 Y$ & 1700V.Y & rTVAT.YA & $r .17$ \\
\hline rᄉ.Ar & 71.18 & OTOVY.A. & T197V.Tr & $r \leqslant 7.0 . \leqslant V$ & $Y . I V$ \\
\hline$M . V$ & 71.94 & $\varepsilon \cdot \Lambda \vee Y_{\text {L. }}$ & 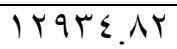 & TVqYA.rV & المتوسط \\
\hline $\begin{array}{c}r \\
(\cdot . \Sigma T V)\end{array}$ & $\begin{array}{c}\cdot 9- \\
(.017)\end{array}$ & $\begin{array}{c}V . r \\
* *(Y) .011)\end{array}$ & $\begin{array}{c}9 . r \\
(\varepsilon .9 \cdot 1)\end{array}$ & $\begin{array}{c}7 . \varepsilon \\
* *(r) .7 \vee 7)\end{array}$ & 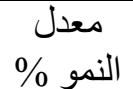 \\
\hline
\end{tabular}

حيث: الأرقام الموجودة بين الأقو اس أسفل قيمة معدل النمو بالجدول الندول تشبير إلى قيمة (F) المحسوبة.

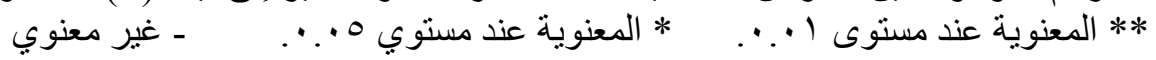

المصدر: جمعت وحسبت من: المعند

- الجهاز المركزي للتعبئة العامة والإحصاء، النشرة السنوية لحركة الإنتاج و التجارة الخارجية و المناح للاستهلاك من السلع الزراعية، القاهرة، أعداد مختلفة.

- الجهاز المركزي للتعبئة العامة والإحصاء، النشرة السنوية لإحصاء الري و المو ارد المائية، القاهرة، أعداد مختلفة.

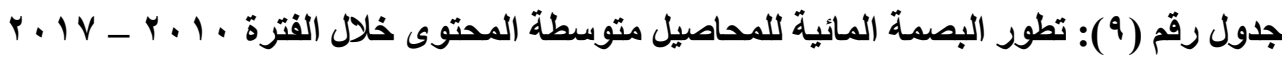

\begin{tabular}{|c|c|c|c|c|c|}
\hline نسبة الاكتفاء الأتي & & \multicolumn{3}{|c|}{ مليون مَّمَ المنئية } & \multirow{2}{*}{ السنوات } \\
\hline المحلية \% & الخارجية \% & الكلية & الخارجية & الاخلية & \\
\hline $0 . Y_{0}$ & $9 \leqslant .10$ & $1.90 \leqslant . \vee 9$ & OVO.Y. & $1 . r \vee 9.09$ & $r .1$. \\
\hline 0.11 & $9 \varepsilon . \wedge Y$ & $9 \leqslant 0 . .11$ & $\varepsilon \wedge 9 . \leqslant \wedge$ & $197 . .74$ & $r .11$ \\
\hline r.q1 & 97.99 & qrVA.YT & ז77.77 & 9.11 .7$. & 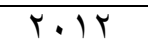 \\
\hline 7.99 & 94.1 & Ir.r7.9人 & $\wedge \varepsilon, \wedge$. & 111147.19 & $r \cdot I r$ \\
\hline 0.01 & $9 \leqslant \leqslant 9$ & $11 T \leqslant \Lambda, Y Y$ & $7 \leqslant 1.90$ & $11 \ldots T . Y V$ & Y.I \\
\hline $0 . V \leqslant$ & $9 \varepsilon . Y 7$ & $1.19 \cdot .71$ & $7 r \leqslant .70$ & 1.17097 & $r .10$ \\
\hline $7.0 \leqslant$ & $94 . \leqslant 7$ & $T r .1 .1 K$ & 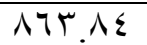 & IKMTV.YA & $r .17$ \\
\hline$V .0$ & $9 Y .90$ & $1 \varepsilon 1 \pi r . \leqslant 9$ & 997.5 & $T \pi \mid r V . \leqslant 0$ & T.IV \\
\hline $0 . V V$ & $9 \leqslant, Y T$ & $11 \leq 7 . \leq 0$ & $T V \leqslant \wedge r$ & $1 . V \wedge 0.7 Y$ & المتو سط \\
\hline $\begin{array}{c}\varepsilon . q \\
(r . \vee \vee \cdot)\end{array}$ & 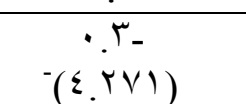 & $\begin{array}{c}\varepsilon .7 \\
*(9.1 T \cdot)\end{array}$ & $=9.0$ & ${ }^{*}(9 . \dot{r} \cdot \cdot)$ & معدل النمو \\
\hline
\end{tabular}

حيث: الأرقام الموجودة بين الأقو اس أسفل قيمة معدل النمو بالجدول تثير إلى قيمة (F) المحسوبة.

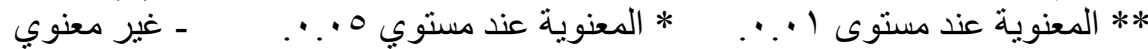

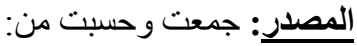

- الجهاز المركزي للتعبئة العامة والإحصاء، النشرة السنوية لحركة الإتتاج و التجارة الخارجية والمتاح للاستهلاك من

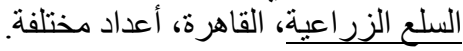

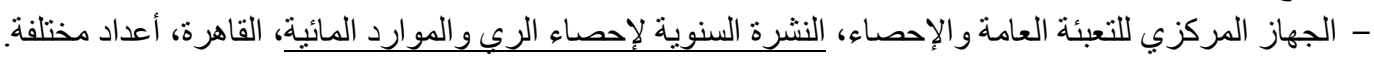

Fayoum J. Agric. Res. \& Dev., Vol. 34, No.2 July, 2020 


\section{Mr}

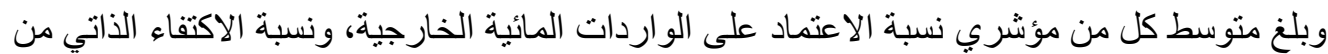

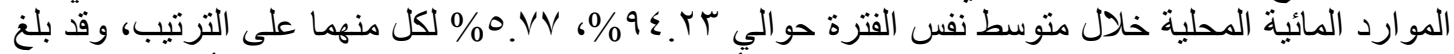

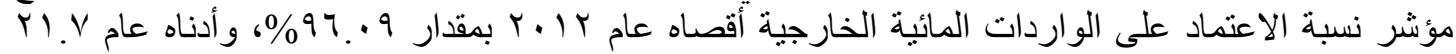

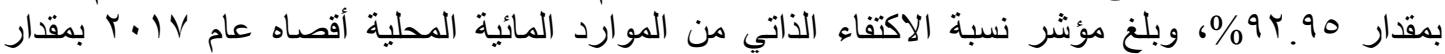

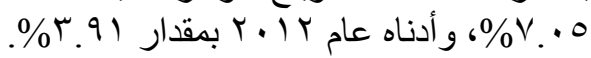

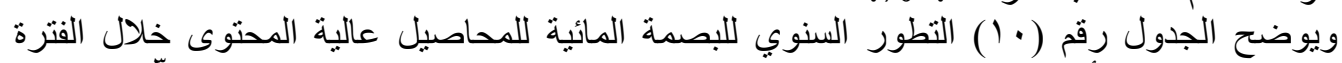

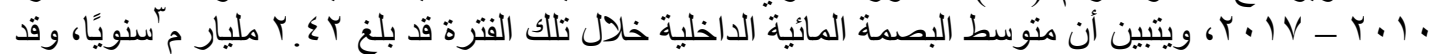

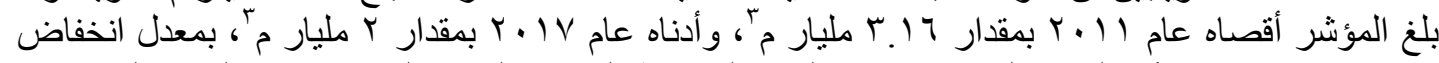

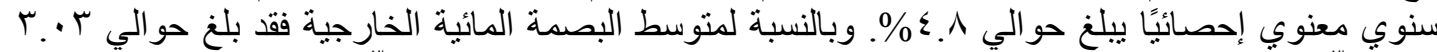

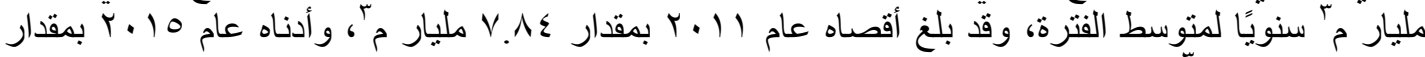

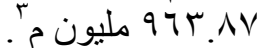

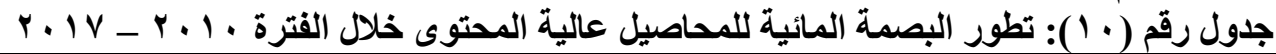

\begin{tabular}{|c|c|c|c|c|c|}
\hline نسبة الاكتفاءع الأاتي منيّ & نسبة الاعتماد على المارداتية & & مليون مَّ/ المدائ & & السنوات \\
\hline$\%$ & الخارجية \%" & الكلية & الخارجية & الاخلية & \\
\hline$\varepsilon r . v \varepsilon$ & $07 . Y 7$ & $\varepsilon \neg \wedge \varepsilon . \wedge 0$ & $r . \leqslant 9 . r)$ & rTro.0 & $r \cdot 1$. \\
\hline$V I . Y \leq$ & YA.VT & 1.991 .99 & VArO.77 & תוז.TIוT & $r+11$ \\
\hline$\leqslant 7.70$ & סTr & EYY 79 & $19 V .90$ & YYOYVE & $r \cdot I r$ \\
\hline TY.Y. & $r v . \wedge$. & 7rov. 7 & r90 10 & $r \leqslant . r . q$. & $r .1 T$ \\
\hline$\xi 1 . \leqslant 1$ & 01.09 & $\varepsilon \varepsilon V Y . \wedge$ & 1101.19 & $r q r \cdot . r q$ & $r \cdot I \leqslant$ \\
\hline rq.0 & $V \cdot . \leqslant 7$ & TYTY.OA & 97T.AV & YYqA.VI & $r .10$ \\
\hline 71.77 & 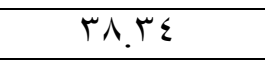 & $0 Y 00 . V \wedge$ & 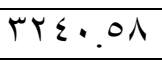 & Y.10.Y. & $r .17$ \\
\hline $0 \leqslant .0 T$ & $\leqslant 0 . \leqslant V$ & $\varepsilon \varepsilon \cdots \varepsilon r$ & rrqq.0 & $r \ldots \wedge q$ & $r \cdot I V$ \\
\hline $01 . r V$ & $\sum \Lambda .7 \mu$ & $0 \leqslant 0 V .17$ & & $r \leqslant Y T . \wedge T$ & المتوسط \\
\hline $\begin{array}{l}1.1- \\
(\cdot .09)\end{array}$ & $\begin{array}{c}1.0 \\
(. .91)\end{array}$ & $\begin{array}{c}7 . r- \\
(1 . r .0\end{array}$ & $\begin{array}{c}V . . \varepsilon- \\
(\cdot .07 \varepsilon)\end{array}$ & $\begin{array}{l}\varepsilon . \Lambda_{-} \\
*(\wedge . q \Gamma \varepsilon)\end{array}$ & النمو \% \\
\hline
\end{tabular}

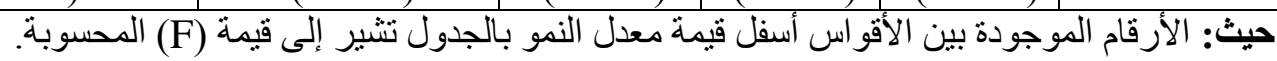

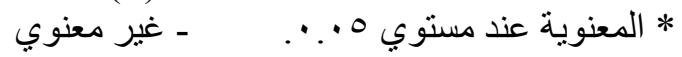

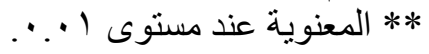

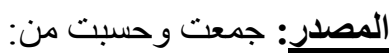
- الجهاز المركزي للتعبئة العامة والإحصاء، النشرة السنوية لحركة الإنتاج و التجارة الخارجية والمتاح للاستهلاك من

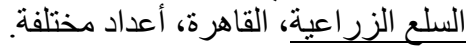

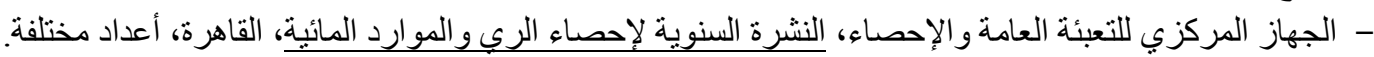

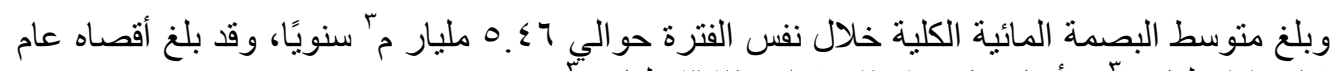

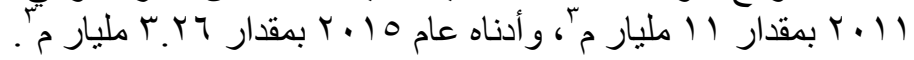

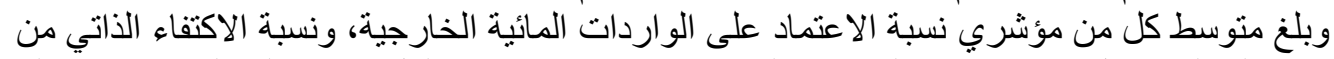

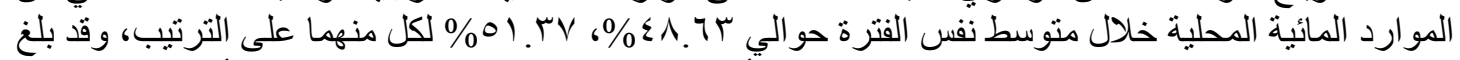

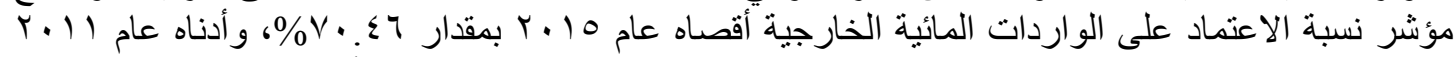

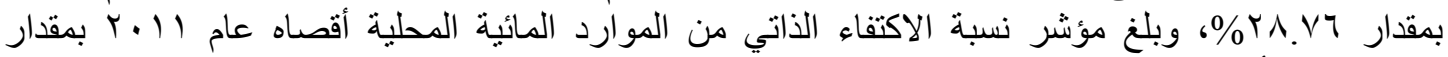

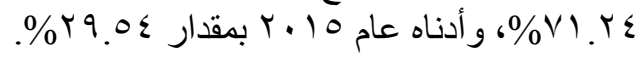

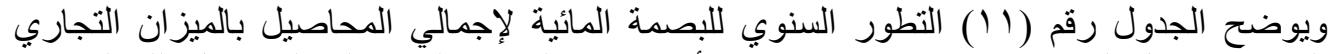

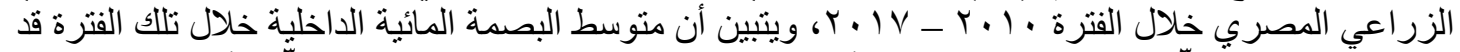

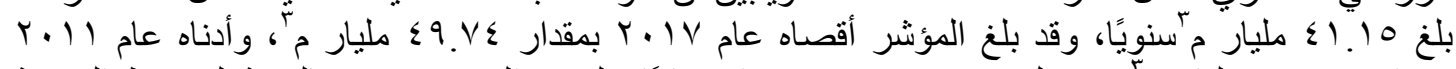

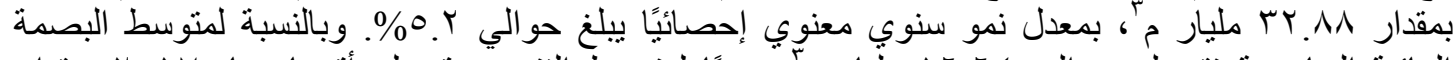

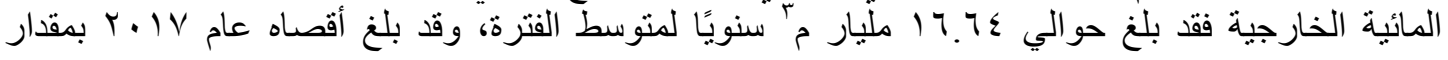

Fayoum J. Agric. Res. \& Dev., Vol. 34, No.2 July, 2020 


\section{Irr}

جr.

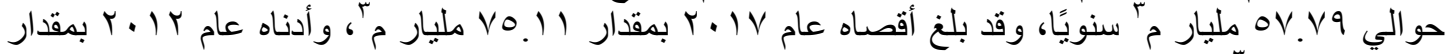

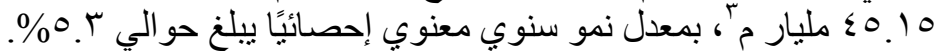

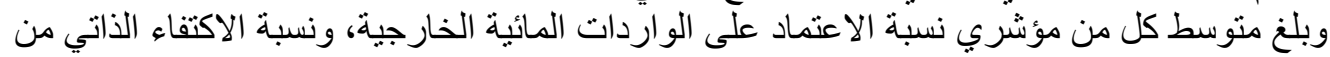

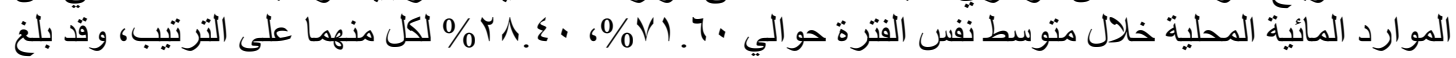

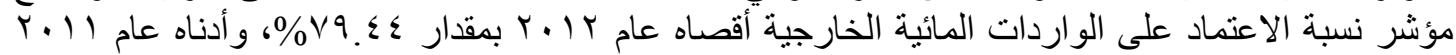

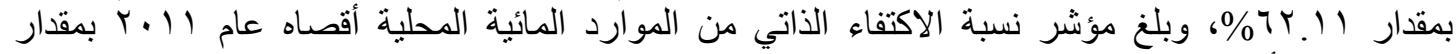

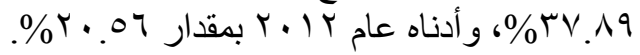

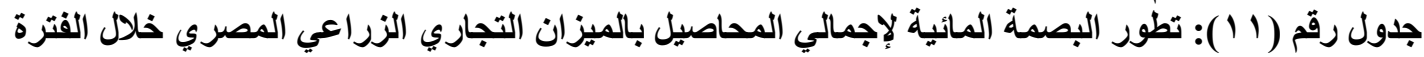
$r$. IV - r. I.

\begin{tabular}{|c|c|c|c|c|c|}
\hline \multirow{2}{*}{ نسبة الاكتفاء الأاتي } & \multirow{2}{*}{ نسبة الاعتماد على الخاردات المائية } & \multicolumn{3}{|c|}{ مليون م آلبصمة المائية } & \multirow[t]{2}{*}{ السنوات } \\
\hline & & الكلية & الخارجية & الاذاخلية & \\
\hline rA. $\cdot 9$ & 71.91 & OY.TI.YA & $1 \leq 747.0 \mathrm{~V}$ & rV & $r \cdot 1$. \\
\hline rV.Aq & 74.11 & orqะr.rq & $r .09 .1 \mathrm{~V}$ & 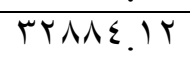 & $r .11$ \\
\hline$r . .07$ & $\vee q . \leqslant \xi$ & $\leqslant 01 \leqslant V . \leqslant r$ & qrAI.YI & TOATt.YY & $r \cdot I r$ \\
\hline TV.rV & VY.TT & OVY90.0 & $1071 . .4 \Lambda$ & $51710 . Y 7$ & $r \cdot I T$ \\
\hline r.r. & VI.V. & $0 \leqslant V \mu Y_{.} \cdot \Lambda$ & IYVOE.AV & $\varepsilon 19 V V . Y_{1}$ & $r \cdot 1 \leqslant$ \\
\hline Y.IV & $V T . \wedge r$ & OTYミI.AT & $1 \leqslant \vee 17 . \leqslant 1$ & \&10Y0. $\leqslant Y$ & r.10 \\
\hline$r \cdot r$ & $79.9 V$ & $\tau \wedge \vee q \vee . \varepsilon r$ & 4.771 .77 & $\varepsilon \Lambda / T_{0} . V V$ & $r .17$ \\
\hline r.VV & 77.YT & .101 .7 .11 & rorgr.qI & 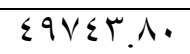 & $r \cdot I V$ \\
\hline r^.乏. & $V 1.7$. & ovVq..V. & $177 \leqslant Y . \wedge 9$ & $\leqslant \| \leqslant \vee . \wedge$ & المتوسط \\
\hline $\begin{array}{c}\cdot . \wedge \\
-(\cdot .70)\end{array}$ & $\begin{array}{c}\cdot T_{-} \\
(\cdot \cdot \mathrm{V})\end{array}$ & $\begin{array}{c}0 . Y \\
(I) . V Y I)\end{array}$ & $\begin{array}{c}7 . r \\
-(1 . \vee 9 \wedge)\end{array}$ & $\begin{array}{c}0 . Y \\
* *(Y 0.0 Y T)\end{array}$ & النمو \%عدل \\
\hline
\end{tabular}

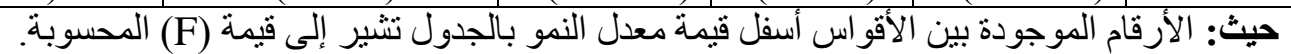

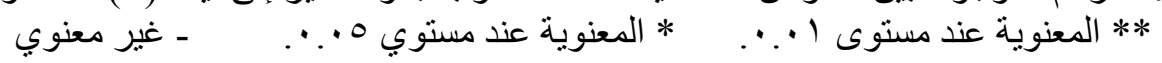

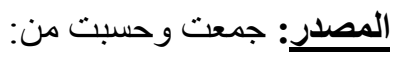

- الجهاز المركزي للتعبئة العامة والإحصاء، النشرة السنوية لحركة الإنتاج و التجارة الخارجية والمتاح للاستهلاك من

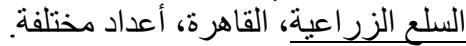

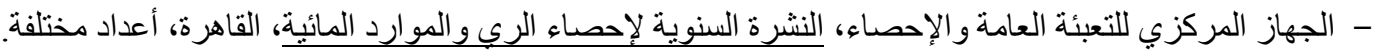
الملخص والتوصياث:

تز ايدت الاحتياجات المائية في مصر بشكل كبير، مع ثبات كمية المورد المائي الأساسي عند 0.00

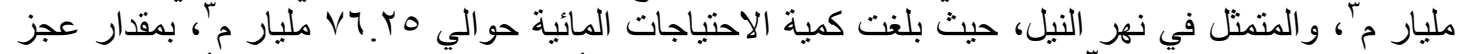

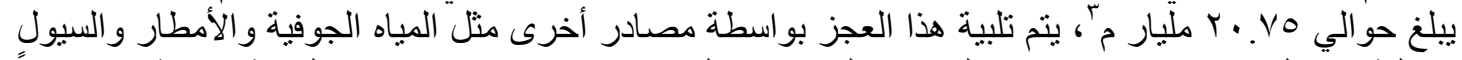

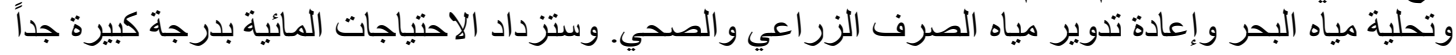

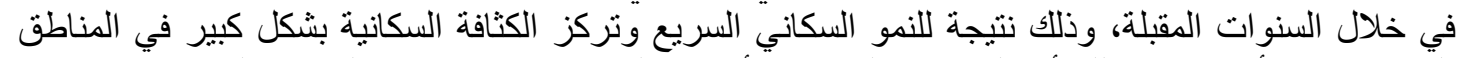

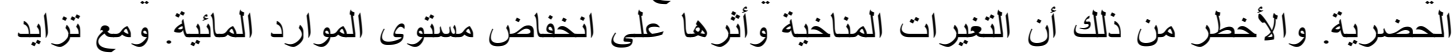

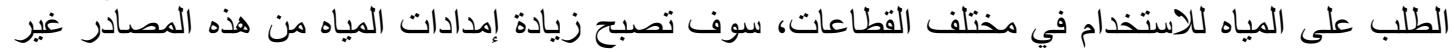

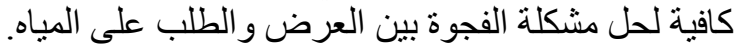

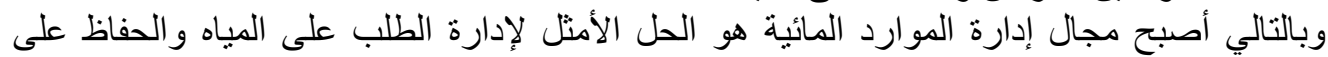
استخدامها. و التي يعد من أهمها الاستفادة من مبدأ المياه الافتر اضية.

يهدف البحث إلى "تحليل أثر المياه الافتراضية على الميزان التجاري الزراعي المصري،، وذلك من خلال

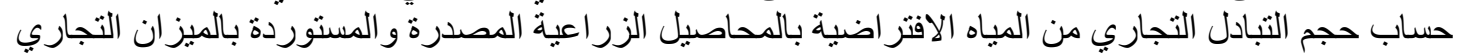

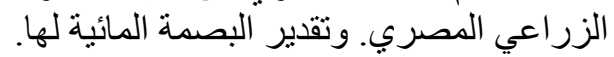

Fayoum J. Agric. Res. \& Dev., Vol. 34, No.2 July, 2020 
$1 \% \varepsilon$

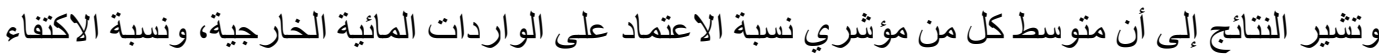

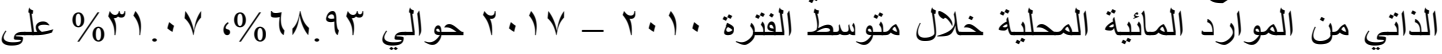

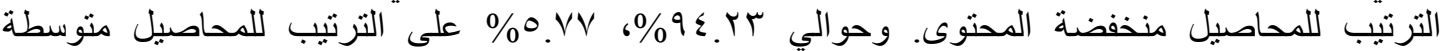

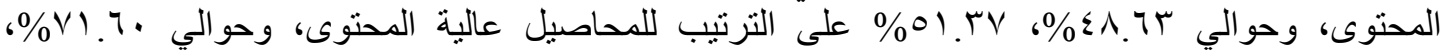

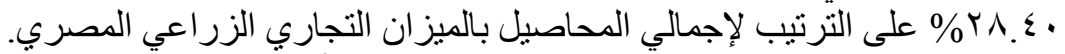

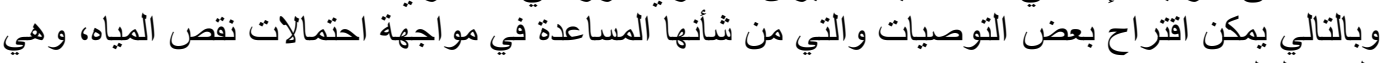

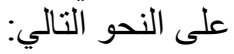

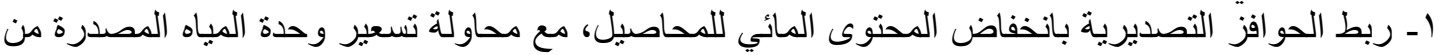

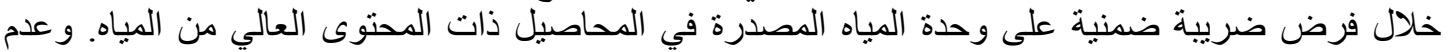

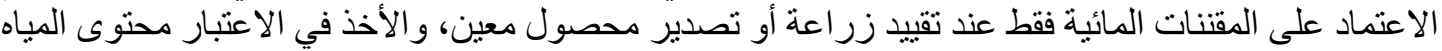
الافتر اضية و عائد وحدة المياه المستهلكة.

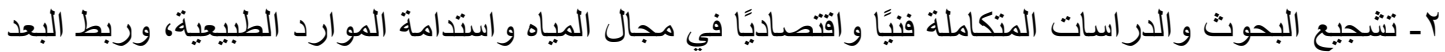

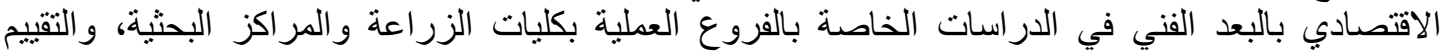

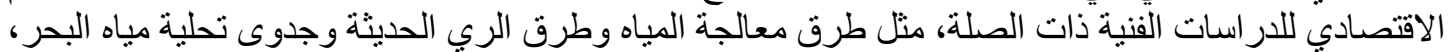

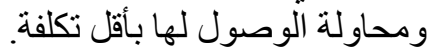

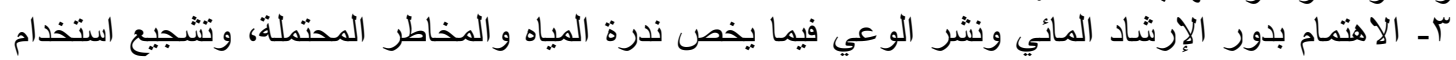

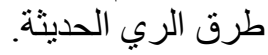

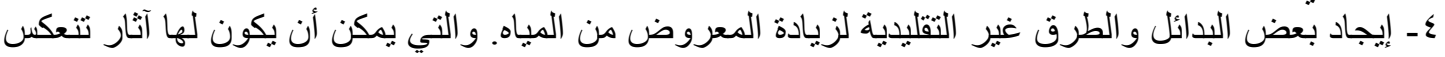

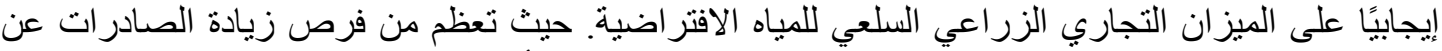

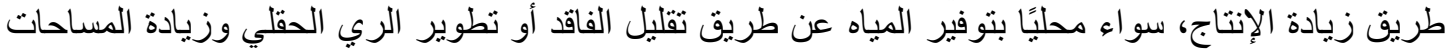

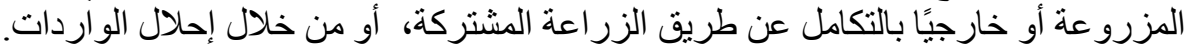

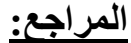

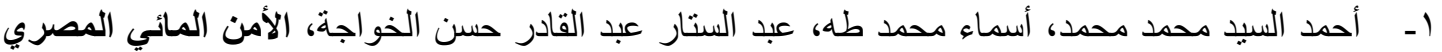

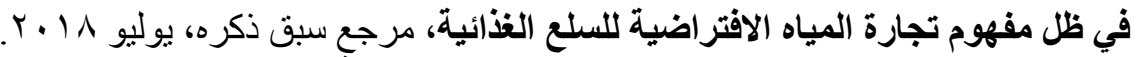

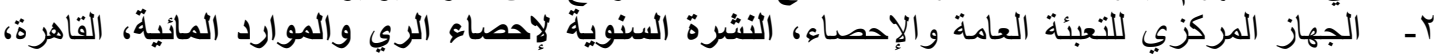
أعداد مختلفة الجري r- الجهاز المركزي للتعبئة العامة والإحصاء، النشرة السنوية لحركة الإنتاج والتجارة الخارجية والمتاح للاستهلاك من السلع الزراعية، القاهرة، أعداد مختلفة.

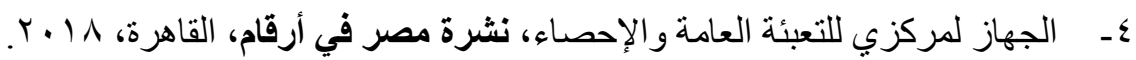

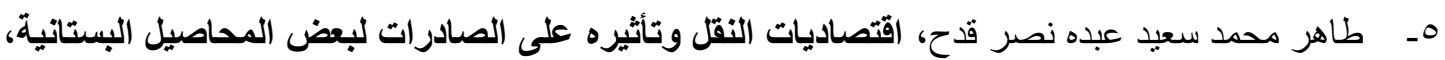

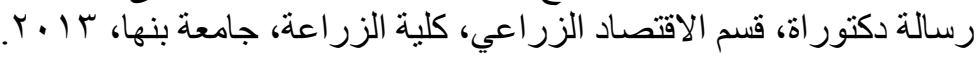

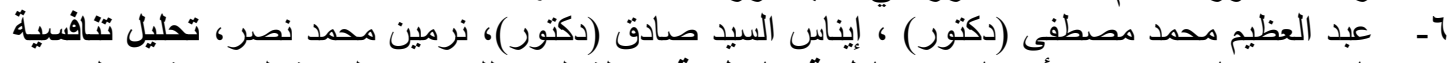
الصادرات المصرين من أهم النباتات الطبية والعطرية، مجلة الفيوم للبحوث و التنمية الزر اعية، مجلد 9 بـ،

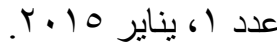

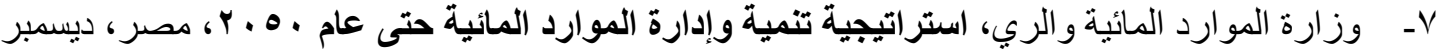
r. 17

8- A. Y. Hoekstra, P. Q. Hung, virtual water trade: a quantification of virtual water flows between nations in relation to international crop trade, value of water research report series no. 11, IHE Delft, Netherlands, September 2002.

Fayoum J. Agric. Res. \& Dev., Vol. 34, No.2 July, 2020 


\section{THE TRADE BALANCE OF VIRTUAL WATER FOR THE MOST IMPORTANT EGYPTIAN AGRICULTURAL CROPS}

\author{
Dr. Abde-Elazeem Mohammed Mostafa \\ Professor of Agricultural Economics* \\ Dr. Waheed Mohamed Elbolony \\ *** Professor of Agricultural Economics
}

ABSTRACT

Water needs have increased dramatically, with the fixed quantity of the main water resource in Egypt at 55.5 billion m3, represented by the Nile River, where the amount of water needs amounted to about 76.25 billion $\mathrm{m} 3$, with a deficit of about 20.75 billion $\mathrm{m} 3$, this deficit is met by other sources. However, with the increasing demand for water for use in various sectors, increasing water supply from these sources will become insufficient to solve the problem of the gap between supply and demand for water.

Water resources management has thus become the ideal solution for managing water demand. One of the most important tools used in demand management is the concept of virtual water.

The research aims to "analyze the impact of virtual water on the Egyptian agricultural trade balance", by calculating the volume of trade exchange from virtual water of exported and imported agricultural crops in the Egyptian agricultural trade balance. And estimate its water footprint.

The results shows that The average of dependence on external water imports and the self-sufficiency ratio of domestic water resources indicators during the average period 2010 - 2017 were about $68.93 \%, 31.07 \%$ for crops with low content of virtual water. And about $94.23 \%, 5.77 \%$ for crops with medium content of virtual water. And $48.63 \%, 51.37$ for crops with high content of virtual water. And $71.60 \%$, $28.40 \%$ for total crops.

${ }^{*}$ Faculty of Agriculture, Fayoum University.

${ }^{* *}$ Agricultural Economic Research Institute, Agricultural Research Center.

Fayoum J. Agric. Res. \& Dev., Vol. 34, No.2 July, 2020 


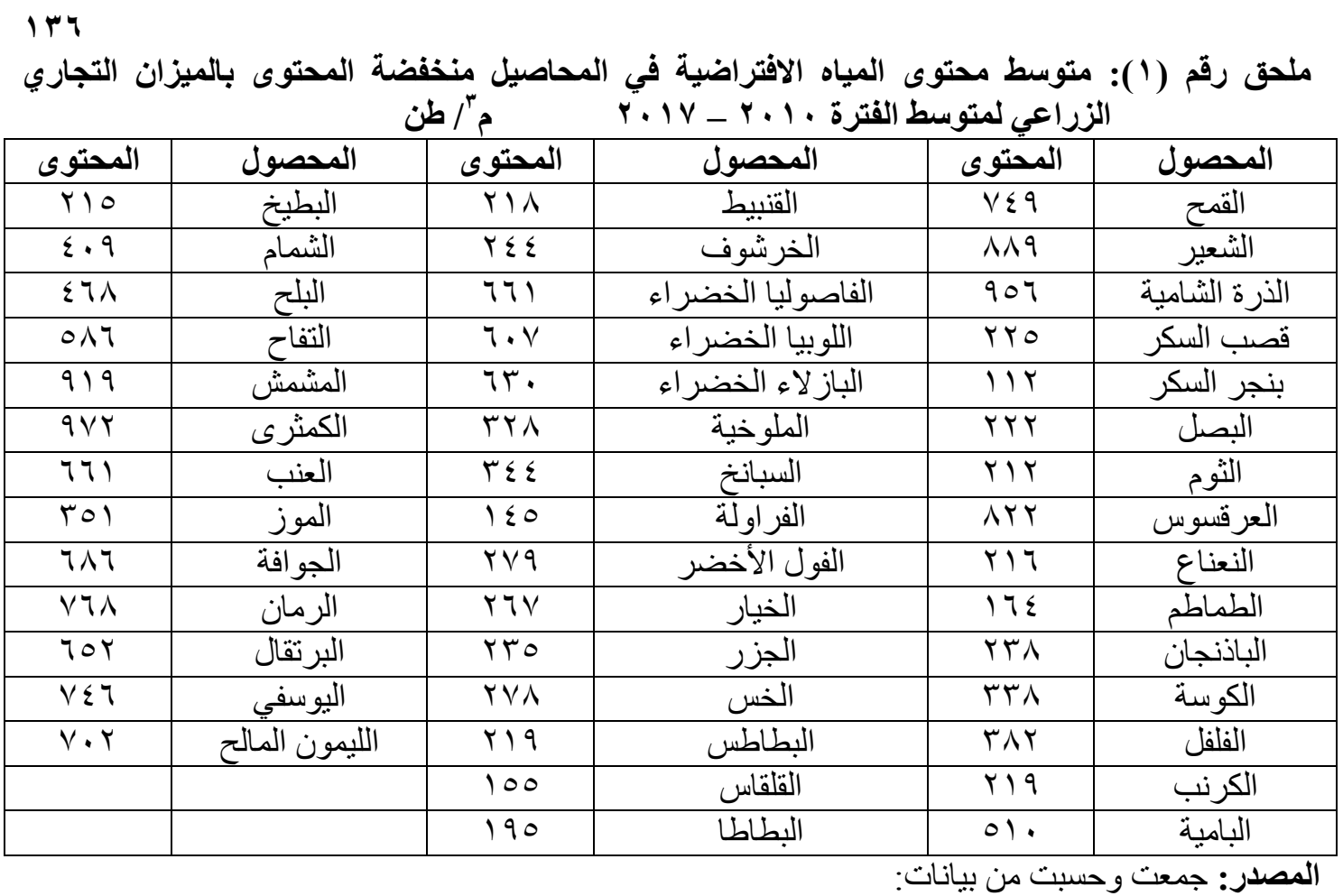

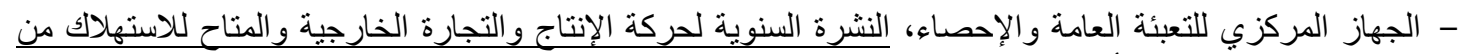

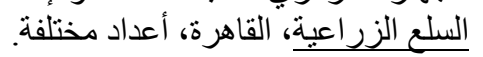
- الجهاز المركزي للتعبئة العامة و الإحصاء، النشرة السنوية لإحصاء الرئه الري والموارد المائية، القاهرة، أعداد مختلفة.

ملحق رقم (r): متوسط محتوى المياه الافتراضية في المحاصيل متوسطة المحتوى بالميزان التجاري

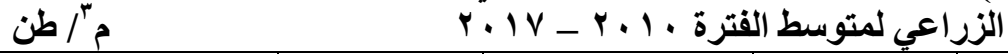

\begin{tabular}{|c|c|c|c|c|c|}
\hline المحتوى & المحصول & المحتوى & المحصول & المحتوى & المحصول \\
\hline 1194 & البرقوق & IVTr & بذرة الكتان & 11497 & الذرة الرفيعة \\
\hline $17 \leqslant 0$ & الزيتون & $11 \wedge r$ & القرنفل & ITrT & الأرز \\
\hline 1798 & المانجو & 1119 & التمر هندي & $1 \cdot \lambda r$ & الفول الجاف \\
\hline \multirow[t]{2}{*}{1 101 } & الليمون الحلو & $170 V$ & الزنجبيل & ITH & العدس \\
\hline & & $11 \wedge \Lambda$ & الخوخ & $100 \leqslant$ & الترمس \\
\hline
\end{tabular}

- الجهاز المركزي للتعبئة العامة والإحصاء، النشرة السنوية لحركة الإنتاج و التجارة الخارجية والمتاح للاستهلاك من

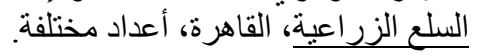
- الجهاز المركزي للتعبئة العامة و الإحصاء، النشرة السنوية لإحصاء الري و الموارد المائية، القاهرة، أعداد مختلفة. 
IrV

ملحق رقم (ب): متوسط محتوى المياه الافتراضية في المحاصيل عالية المحتوى بالميزان التجاري الزراعي

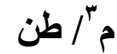

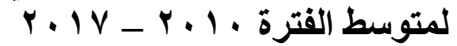

\begin{tabular}{|c|c|c|c|c|c|}
\hline المحتوى & المحصول & المحتوى & المحصول & المحتوى & المحصول \\
\hline$r \leqslant V T$ & الثمر & YYVY & عباد الشمس & TYTY & الحلبة \\
\hline$r \sum V T$ & الحناء & $\varepsilon \cdot \Gamma \xi$ & اليانسون & rाI & الحمص \\
\hline 9949 & الكركديه & אי & الكسبرة الجافة & rYq. & الفاصوليا الجافة \\
\hline rYOQ & التين & $01 \ldots$ & الكمون & T\&. & اللوبيا الجافة \\
\hline rqrq & اللوز & $0 \leqslant V Y$ & الكر اوية & TYOT & الباز لاء الجافة \\
\hline$\sum 911$ & الجوز & VTro & الفلفل الأسود & 1.01 & بذرة القطن \\
\hline $0 Y 01$ & البندق & $100 Y 7$ & القرفة & rAYA & الفول السوداني \\
\hline \multirow[t]{2}{*}{$r \cdot V I$} & الفستق & TYNI & شيح البابونج & $r \leqslant 9 \wedge$ & فول الصويا \\
\hline & & YIYI & البردقوش & OTVY & السمسم \\
\hline
\end{tabular}

- الجهاز المركزي للتعبئة العامة والإحصاء، النشرة السنوية لحركة الإنتاج و التجارة الخارجية والمتاح للاستهلاك من

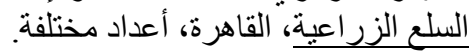

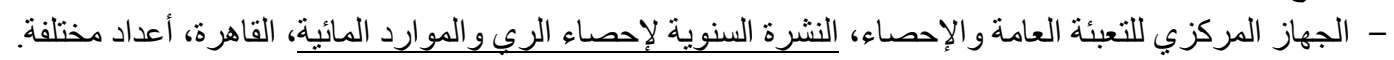

Fayoum J. Agric. Res. \& Dev., Vol. 34, No.2 July, 2020 